\title{
A high-order time formulation of the RBC schemes for unsteady compressible Euler equations
}

\author{
A. Lerat \\ DynFluid Lab., Arts et Metiers ParisTech, 151 Boulevard de l'Hopital, 75013 Paris, France
}

\begin{abstract}
Residual-Based Compact (RBC) schemes can approximate the compressible Euler equations with a high space-accuracy on a very compact stencil. For instance on a 2-D Cartesian mesh, the 5th and 7th-order accuracy can be reached on a 5x5-point stencil. The time integration of the RBC schemes uses a fully implicit method of 2nd-order accuracy (Gear method) usually solved by a dual-time approach. This method is efficient for computing compressible flows in slow unsteady regimes, but for quick unsteady flows, it may be costly and not accurate enough. A new time-formulation is proposed in the present paper. Unusually, in a RBC scheme the time derivative occurs, through linear discrete operators due to compactness, not only in the main residual but also in the other two residuals (in 2-D) involved in the numerical dissipation. To extract the time derivative, a space-factorization method which preserves the high accuracy in space is developed for reducing the algebra to the direct solution of simple linear systems on the mesh lines. Then a time-integration of high accuracy is selected for the RBC schemes by comparing the efficiency of four classes of explicit methods. The new time-formulation is validated for the diagonal advection of a Gaussian shape, the rotation of a hump, the advection of a vortex for a long time and the interaction of a vortex with a shock.
\end{abstract}

Keywords: Residual-Based Compact scheme, High order, Time integration, Unsteady compressible flows

\section{Introduction}

Most numerical schemes for compressible flow simulations on a structured mesh are based on a directional approach in which space derivatives are approximated independently direction by direction. In contrast, residual-based compact $(R B C)$ schemes are constructed from the complete residual $r$ containing all the terms in the governing equations including the time derivative. In such a scheme, the numerical dissipation as well as the consistent part is expressed only in terms of approximations of $r$. More precisely, the numerical dissipation involves space first-derivatives of $r$. Several approximations of $r$ are used in a $R B C$ scheme. All are compact and deduced from Pade fractions of discrete operators after eliminating the denominators. On a Cartesian mesh, $R B C$ schemes can approximate a hyperbolic system of conservation laws in $d$-dimension with a 5 th or 7 th-order accuracy on a $5^{d}$-point stencil. For these odd accuracy-orders, the leading error (of order 5 or 7 ) is dissipative and dominates the dispersive error (of order 6 or 8), which is a favorable feature for robustness. Description and analysis of the $R B C$ schemes can be found in [1-6]. A related approach developed on unstructured meshes concerns the residual-distribution schemes of Abgrall, Deconinck and Ricchiuto [7-10] for which the residuals are distributed to the nodes of triangles or tetrahedrons.

The $R B C$ schemes have been developed so far with a time-formulation based on the Gear method. The resulting method is efficient for computing compressible flows in steady and slow unsteady regimes. However, it requires an iterative method to advance the solution (dual-time approach or Newton sub-iterations) and its time accuracy is limited to order 2 , which is not sufficient for some applications. This is the reason why

Email address: alain.lerat@ensam.eu (A. Lerat) 
we investigate a new time-formulation of high accuracy. A specific issue is that a $R B C$ scheme contains the time derivative $\partial w / \partial t$ at several places through linear discrete operators due to compactness. In $d$ dimension, $\partial w / \partial t$ occurs $d$ times in the numerical dissipation and once in the consistent part of the scheme. We first assess a simplification of estimating the time derivatives in dissipation from a previous step. Then we investigate a rigorous extraction of the time derivative. Using a method of space factorization with correction, we extract $\partial w / \partial t$ by solving simple linear algebraic systems on the mesh lines, while preserving the high order in space. Finally we discuss the choice of a high-order time-integration. We compare the efficiency of four classes of time approximation: the Adams-Bashforth methods, the extrapolated Backward Differential Formulas, explicit combinations of Adams-Bashforth and Adams-Moulton methods $(A B M)$ and the Runge-Kutta methods. The computing time depends on two crucial factors: $i$ ) the number of calculations of flux balance and extraction of $\partial w / \partial t$ and $i i)$ the magnitude of the maximal time-step allowed for stability. For a long time-integration at fourth order of a two-dimensional Euler problem, the $A B M$ method is found to be the least expensive in CPU-time.

The paper is organized as follows. Section 2 reminds the concept of residual-based scheme and the compact space-approximation for solving a hyperbolic system of conservation laws. $R B C$ schemes of order 3,5 and 7 are presented. Section 3 describes the new time-formulation of the $R B C$ schemes. Section 4 validates the new formulation on several test-problems: the diagonal advection of a Gaussian shape, the rotation of a hump, the advection of a vortex for a long time and the interaction of a shock with a vortex. Conclusions are drawn and further work is planned in Section 5.

\section{RBC space approximation}

\subsection{Concept of residual-based scheme}

Consider the hyperbolic system of conservation laws:

$$
\frac{\partial w}{\partial t}+\frac{\partial f}{\partial x}+\frac{\partial g}{\partial y}=0
$$

where $t$ is the time, $x$ and $y$ are Cartesian space coordinates, $w$ is the state vector and $f=f(w), g=g(w)$ are flux components depending smoothly on $w$. The Jacobian matrices of the flux are denoted $A=\mathrm{d} f / \mathrm{d} w$ and $B=\mathrm{d} g / \mathrm{d} w$.

System (1) is approximated in space on a uniform mesh $\left(x_{j}=j \delta x, y_{k}=k \delta y\right)$ with steps $\delta x$ and $\delta y$ of the same order of magnitude, say $\mathcal{O}(h)$, using a residual-based-compact $(R B C)$ scheme. Such a scheme is a compact discrete form of

$$
\frac{\partial w}{\partial t}+\frac{\partial f}{\partial x}+\frac{\partial g}{\partial y}=\frac{\delta x}{2} \frac{\partial}{\partial x}\left[\Phi_{1}\left(\frac{\partial w}{\partial t}+\frac{\partial f}{\partial x}+\frac{\partial g}{\partial y}\right)\right]+\frac{\delta y}{2} \frac{\partial}{\partial y}\left[\Phi_{2}\left(\frac{\partial w}{\partial t}+\frac{\partial f}{\partial x}+\frac{\partial g}{\partial y}\right)\right]
$$

where $\Phi_{1}$ and $\Phi_{2}$ are matrices depending only on the eigensystems of the Jacobian matrices $A$ and $B$ and on the step ratio $\delta x / \delta y$. They use no tuning parameters or limiters. More precisely, let $T_{A}$ (respectively $T_{B}$ ) be a matrix the columns of which are the right eigenvectors of $A$ (resp. $B$ ) and let $a^{(i)}\left(\right.$ resp. $\left.b^{(i)}\right)$ be the eigenvalues of $A$ (resp. $B$ ), matrices $\Phi_{1}$ and $\Phi_{2}$ are defined as

with

$$
\Phi_{1}=T_{A} \operatorname{Diag}\left[\phi_{1}^{(i)}\right] T_{A}^{-1}, \quad \Phi_{2}=T_{B} \operatorname{Diag}\left[\phi_{2}^{(i)}\right] T_{B}^{-1}
$$

$$
\begin{array}{r}
\phi_{1}^{(i)}=\operatorname{sgn}\left(a^{(i)}\right) \phi^{(i)}, \quad \phi_{2}^{(i)}=\operatorname{sgn}\left(b^{(i)}\right) \psi^{(i)} \\
\phi^{(i)}=\min \left(1, \frac{\delta y\left|a^{(i)}\right|}{\delta x \mathrm{~m}(B)}\right), \quad \psi^{(i)}=\min \left(1, \frac{\delta x\left|b^{(i)}\right|}{\delta y \mathrm{~m}(A)}\right)
\end{array}
$$

where Diag $\left[d^{(i)}\right]$ denotes a diagonal matrix with diagonal entries $d^{(i)}$ and $\mathrm{m}(A)=\min _{i}\left|a^{(i)}\right|, \operatorname{m}(B)=\min _{i}\left|b^{(i)}\right|$. Equation (2) can also be written as

$$
r=\frac{1}{2}\left[\delta x \frac{\partial}{\partial x}\left(\Phi_{1} r\right)+\delta y \frac{\partial}{\partial y}\left(\Phi_{2} r\right)\right]
$$


with the exact residual

$$
r=\frac{\partial w}{\partial t}+\frac{\partial f}{\partial x}+\frac{\partial g}{\partial y}
$$

To describe the space approximation of (3), we introduce basic discrete operators over one mesh interval in each space direction:

$$
\begin{aligned}
\left(\delta_{1} v\right)_{j+\frac{1}{2}, k}=v_{j+1, k}-v_{j, k} & \left(\delta_{2} v\right)_{j, k+\frac{1}{2}}=v_{j, k+1}-v_{j, k} \\
\left(\mu_{1} v\right)_{j+\frac{1}{2}, k}=\frac{1}{2}\left(v_{j+1, k}+v_{j, k}\right) & \left(\mu_{2} v\right)_{j, k+\frac{1}{2}}=\frac{1}{2}\left(v_{j, k+1}+v_{j, k}\right)
\end{aligned}
$$

where $j$ and $k$ are integers or half integers. All these discrete operators commute. For instance:

$$
\begin{gathered}
\left(\frac{\delta_{1} \mu_{1} f}{\delta x}\right)_{j, k}=\left(\frac{\mu_{1} \delta_{1} f}{\delta x}\right)_{j, k}=\frac{f_{j+1, k}-f_{j-1, k}}{2 \delta x} \\
\left(\delta_{1}^{2} f\right)_{j, k}=\left(\delta_{1}\left(\delta_{1} f\right)\right)_{j, k}=f_{j+1, k}-2 f_{j, k}+f_{j-1, k}
\end{gathered}
$$

Using different compact centered approximations $\tilde{r}_{j, k},\left(\tilde{r}_{1}\right)_{j+\frac{1}{2}, k}$ and $\left(\tilde{r}_{2}\right)_{j, k+\frac{1}{2}}$ in Eq.(3), we obtain the residual-based compact scheme:

$$
\tilde{r}_{j, k}=\frac{1}{2}\left[\delta_{1}\left(\Phi_{1} \tilde{r}_{1}\right)+\delta_{2}\left(\Phi_{2} \tilde{r}_{2}\right)\right]_{j, k}
$$

The right-hand side of (4) is a numerical dissipation. Despite appearance, this dissipation is not simply of order one because $\left(\tilde{r}_{1}\right)$ and $\left(\tilde{r}_{2}\right)$ approximate the exact residual $r=0$. Clearly, if $\tilde{r}_{j, k}$ approximates $r$ at order $2 p$ and $\left(\tilde{r}_{1}\right)_{j+\frac{1}{2}, k},\left(\tilde{r}_{2}\right)_{j, k+\frac{1}{2}}$ approximate $r$ at order $2 p-2$, then the scheme (4) is accurate at order $2 p-1$ in space and denoted as $R B C_{2 p-1}$. In such a scheme, the dissipation error (of order $2 p-1$ ) dominates the dispersive error (of order $2 p$ ), which is a good feature for robustness.

More details on the numerical dissipation and the choice of the matrices $\left(\Phi_{1}\right)_{j+\frac{1}{2}, k}$ and $\left(\Phi_{2}\right)_{j, k+\frac{1}{2}}$ can be found in $[1,3,5]$. Spectral properties of the scheme are described in $[6]$.

\subsection{General form of the residuals for a RBC scheme on a $5 \times 5$-point-stencil}

Compact schemes for compressible flows have been mainly developed as centered approximations in space (see [11-14] for instance) relying on the use of artificial viscosities, numerical filters or limiters for shock capturing. Upwind compact schemes have also been proposed in $[15,16]$. The present RBC scheme uses three compact centered-approximations of the residual and relies only on its internal numerical dissipation. On a $5 \times 5$-point stencil, the main residual, i.e. the left-hand side of (4), takes the general compact form (see $[3-5])$ :

$$
\tilde{r}=\left(I+\bar{b} \delta_{1}^{2}+\bar{c} \delta_{1}^{4}\right)\left(I+\bar{b} \delta_{2}^{2}+\bar{c} \delta_{2}^{4}\right) \frac{\partial w}{\partial t}+\left(I+\bar{b} \delta_{2}^{2}+\bar{c} \delta_{2}^{4}\right)\left(I+\bar{a} \delta_{1}^{2}\right) \frac{\delta_{1} \mu_{1} f}{\delta x}+\left(I+\bar{b} \delta_{1}^{2}+\bar{c} \delta_{1}^{4}\right)\left(I+\bar{a} \delta_{2}^{2}\right) \frac{\delta_{2} \mu_{2} g}{\delta y}
$$

where $I$ is the identity operator and the subscripts $j, k$ are omitted. This residual depends on the three scalar coefficients $\bar{a}, \bar{b}$ and $\bar{c}$. It is at least second-order accurate, but can be much more accurate with a suitable choice of the coefficients. Namely, $\tilde{r}$ is accurate

- at least at fourth-order iff:

$$
\bar{b}-\bar{a}=\frac{1}{6}
$$

- at least at sixth-order iff in addition:

$$
\frac{1}{6} \bar{b}-\bar{c}=\frac{1}{30}
$$

- at eighth-order iff in addition:

$$
\frac{1}{30} \bar{b}-\frac{1}{6} \bar{c}=\frac{1}{140}
$$


To obtain a $R B C$ scheme on a $5 \times 5$-point stencil, the residuals in the dissipation $\left(\tilde{r}_{1}\right)_{j+\frac{1}{2}, k}$ and $\left(\tilde{r}_{2}\right)_{j, k+\frac{1}{2}}$ should use no more than $4 \times 5$ and $5 \times 4$ points, respectively. Their general forms are:

$$
\begin{aligned}
& \tilde{r}_{1}=\left(I+b \delta_{2}^{2}+c \delta_{2}^{4}\right)\left[\left(I+a^{\mu} \delta_{1}^{2}\right) \mu_{1} \frac{\partial w}{\partial t}+\left(I+a^{\delta} \delta_{1}^{2}\right) \frac{\delta_{1} f}{\delta x}\right]+\left(I+a^{\mu} \delta_{1}^{2}\right)\left(I+a \delta_{2}^{2}\right) \frac{\delta_{2} \mu_{2} \mu_{1} g}{\delta y} \\
& \tilde{r}_{2}=\left(I+b \delta_{1}^{2}+c \delta_{1}^{4}\right)\left[\left(I+a^{\mu} \delta_{2}^{2}\right) \mu_{2} \frac{\partial w}{\partial t}+\left(I+a^{\delta} \delta_{2}^{2}\right) \frac{\delta_{2} g}{\delta y}\right]+\left(I+a^{\mu} \delta_{2}^{2}\right)\left(I+a \delta_{1}^{2}\right) \frac{\delta_{1} \mu_{1} \mu_{2} f}{\delta x} .
\end{aligned}
$$

Residuals $\tilde{r}_{1}$ and $\tilde{r}_{2}$ depend on the five scalar coefficients $a, b, c, a^{\mu}$ and $a^{\delta}$. They are accurate at least at second-order and

- at least at fourth-order iff:

- at sixth-order iff in addition:

$$
b-a=\frac{1}{6}, \quad a^{\delta}-a^{\mu}=\frac{1}{12}
$$

$$
\frac{1}{6} b-c=\frac{1}{30}, \quad a^{\mu}=\frac{1}{10}
$$

\subsection{Choice of the scheme coefficients}

- By satisfying condition (6) only, we obtain $\tilde{r}=\mathcal{O}\left(h^{4}\right), \tilde{r}_{1}=\mathcal{O}\left(h^{2}\right), \tilde{r}_{2}=\mathcal{O}\left(h^{2}\right)$ and the scheme (4) is third-order accurate. This can be achieved on a $3 \times 3$-point stencil by cancelling $\bar{a}$ and $\bar{c}$ in the main residual (5) and $a, c, a^{\mu}$ and $a^{\delta}$ in the residuals in the dissipation (9), the latter using $2 \times 3$ and $3 \times 2$ points only. Therefore, the third-order schemes on a 3x3-point stencil are defined by:

$$
\bar{a}=0, \quad \bar{b}=\frac{1}{6}, \quad \bar{c}=0, \quad a=0, \quad c=0, \quad a^{\mu}=0, \quad a^{\delta}=0
$$

and $b$ is free.

- By satisfying conditions (6), (7) and (10), we obtain $\tilde{r}=\mathcal{O}\left(h^{6}\right), \tilde{r}_{1}=\mathcal{O}\left(h^{4}\right), \tilde{r}_{2}=\mathcal{O}\left(h^{4}\right)$ and the scheme is fifth-order accurate. This cannot be achieved on a 3x3-point stencil, but it is possible to get fifth-order schemes for which the operators acting on the time-derivative $\partial w / \partial t$ involve 3 points only in each space direction. This is done by cancelling $\bar{c}, c$ and $a^{\mu}$, which gives:

$$
\bar{a}=\frac{1}{30}, \quad \bar{b}=\frac{1}{5}, \quad \bar{c}=0, \quad a=b-\frac{1}{6}, \quad c=0, \quad a^{\mu}=0, \quad a^{\delta}=\frac{1}{12}
$$

where $b$ is free. Such schemes with 3 -point operators on $\partial w / \partial t$ will be interesting for our new timeformulation.

- By satisfying conditions $(6),(7),(8),(10)$ and (11), we obtain $\tilde{r}=\mathcal{O}\left(h^{8}\right), \tilde{r}_{1}=\mathcal{O}\left(h^{6}\right), \tilde{r}_{2}=\mathcal{O}\left(h^{6}\right)$ and the scheme is seventh-order accurate with coefficients:

$$
\bar{a}=\frac{5}{42}, \quad \bar{b}=\frac{2}{7}, \quad \bar{c}=\frac{1}{70}, \quad a=6 c+\frac{1}{30}, \quad b=6 c+\frac{1}{5}, \quad a^{\mu}=\frac{1}{10}, \quad a^{\delta}=\frac{11}{60}
$$

where $c$ is free.

\subsection{Schemes satisfying the $\chi$ criterion}

The effective dissipation induced by the numerical dissipation term

$$
\tilde{d}=\frac{1}{2}\left[\delta_{1}\left(\Phi_{1} \tilde{r}_{1}\right)+\delta_{2}\left(\Phi_{2} \tilde{r}_{2}\right)\right]
$$

has been identified and analyzed in [5]. For any $R B C_{2 p-1}$ scheme, a Taylor expansion of (15) gives:

$$
\tilde{d}=(-1)^{p-1} \kappa\left\{\frac{\partial}{\partial x}\left[\Phi_{1}\left(\delta x^{2 p-1} \frac{\partial^{2 p-1} f}{\partial x^{2 p-1}}+\chi \delta x \delta y^{2 p-2} \frac{\partial^{2 p-1} g}{\partial x^{2 p-1}}\right)\right]+\frac{\partial}{\partial y}\left[\Phi_{2}\left(\chi \delta x^{2 p-2} \delta y \frac{\partial^{2 p-1} f}{\partial x^{2 p-1}}+\delta y^{2 p-1} \frac{\partial^{2 p-1} g}{\partial x^{2 p-1}}\right)\right]\right\}
$$


where $\kappa>0$ and $\chi$ are two constant coefficients depending on the scheme. Note that (16) does not contain time derivatives since they have been replaced by space derivatives using the exact system (1).

For the third-order schemes on a $3 \times 3$-point stencil, coefficients $\kappa$ and $\chi$ are:

$$
\kappa=\frac{1}{24}, \quad \chi=12\left(b-\frac{1}{6}\right)
$$

For fifth-order schemes, they are:

$$
\kappa=\frac{1}{24}\left(\frac{1}{10}-a^{\mu}\right), \quad \chi=\frac{1}{2 \kappa}\left(\frac{b}{6}-c-\frac{1}{30}\right)
$$

In particular, for the fifth-order schemes with 3-point operators on $\partial w / \partial t$ :

$$
\kappa=\frac{1}{240}, \quad \chi=20\left(b-\frac{1}{5}\right)
$$

For seventh-order schemes, we have:

$$
\kappa=\frac{1}{5600}, \quad \chi=\frac{280}{3}\left(c-\frac{1}{70}\right)
$$

It has been proved in [5] that any $R B C_{2 p-1}$ scheme should meet the $\chi$-criterion for the differential operator in (16) to be dissipative for a general multidimensional problem. This criterion simply requires that $\chi=0$. In other words, crossed derivatives are present in the right-hand side of the continuous formulation (2), but no crossed derivative should appear in the differential operator (16) induced by the discretization. The stabilizing effect of the $\chi$-criterion on skew waves has been confirmed by numerical experiments [5]. Taking into account the $\chi$-criterion, we obtain $b=1 / 6$ in (12), $b=1 / 5$ in (13), $c=1 / 70$ in (14) and thus a unique scheme for each odd order of accuracy. The residuals of the corresponding schemes are listed below.

- Residuals for the $R B C_{3}$ scheme:

$$
\begin{gathered}
\tilde{r}=\left(I+\frac{1}{6} \delta_{1}^{2}\right)\left(I+\frac{1}{6} \delta_{2}^{2}\right) \frac{\partial w}{\partial t}+\left(I+\frac{1}{6} \delta_{2}^{2}\right) \frac{\delta_{1} \mu_{1} f}{\delta x}+\left(I+\frac{1}{6} \delta_{1}^{2}\right) \frac{\delta_{2} \mu_{2} g}{\delta y} \\
\tilde{r}_{1}=\left(I+\frac{1}{6} \delta_{2}^{2}\right)\left(\mu_{1} \frac{\partial w}{\partial t}+\frac{\delta_{1} f}{\delta x}\right)+\frac{\delta_{2} \mu_{2} \mu_{1} g}{\delta y} \\
\tilde{r}_{2}=\left(I+\frac{1}{6} \delta_{1}^{2}\right)\left(\mu_{2} \frac{\partial w}{\partial t}+\frac{\delta_{2} g}{\delta y}\right)+\frac{\delta_{1} \mu_{1} \mu_{2} f}{\delta x}
\end{gathered}
$$

- Residuals for the $R_{B} C_{5}$ scheme:

$$
\begin{gathered}
\tilde{r}=\left(I+\frac{1}{5} \delta_{1}^{2}\right)\left(I+\frac{1}{5} \delta_{2}^{2}\right) \frac{\partial w}{\partial t}+\left(I+\frac{1}{5} \delta_{2}^{2}\right)\left(I+\frac{1}{30} \delta_{1}^{2}\right) \frac{\delta_{1} \mu_{1} f}{\delta x}+\left(I+\frac{1}{5} \delta_{1}^{2}\right)\left(I+\frac{1}{30} \delta_{2}^{2}\right) \frac{\delta_{2} \mu_{2} g}{\delta y} \\
\tilde{r}_{1}=\left(I+\frac{1}{5} \delta_{2}^{2}\right)\left[\mu_{1} \frac{\partial w}{\partial t}+\left(I+\frac{1}{12} \delta_{1}^{2}\right) \frac{\delta_{1} f}{\delta x}\right]+\left(I+\frac{1}{30} \delta_{2}^{2}\right) \frac{\delta_{2} \mu_{2} \mu_{1} g}{\delta y} \\
\tilde{r}_{2}=\left(I+\frac{1}{5} \delta_{1}^{2}\right)\left[\mu_{2} \frac{\partial w}{\partial t}+\left(I+\frac{1}{12} \delta_{2}^{2}\right) \frac{\delta_{2} g}{\delta y}\right]+\left(I+\frac{1}{30} \delta_{1}^{2}\right) \frac{\delta_{1} \mu_{1} \mu_{2} f}{\delta x}
\end{gathered}
$$

- Residuals for the $R B C_{7}$ scheme:

$$
\begin{aligned}
\tilde{r}= & \left(I+\frac{2}{7} \delta_{1}^{2}+\frac{1}{70} \delta_{1}^{4}\right)\left(I+\frac{2}{7} \delta_{2}^{2}+\frac{1}{70} \delta_{2}^{4}\right) \frac{\partial w}{\partial t} \\
& +\left(I+\frac{2}{7} \delta_{2}^{2}+\frac{1}{70} \delta_{2}^{4}\right)\left(I+\frac{5}{42} \delta_{1}^{2}\right) \frac{\delta_{1} \mu_{1} f}{\delta x}+\left(I+\frac{2}{7} \delta_{1}^{2}+\frac{1}{70} \delta_{1}^{4}\right)\left(I+\frac{5}{42} \delta_{2}^{2}\right) \frac{\delta_{2} \mu_{2} g}{\delta y}
\end{aligned}
$$




$$
\begin{aligned}
& \tilde{r}_{1}=\left(I+\frac{2}{7} \delta_{2}^{2}+\frac{1}{70} \delta_{2}^{4}\right)\left[\left(I+\frac{1}{10} \delta_{1}^{2}\right) \mu_{1} \frac{\partial w}{\partial t}+\left(I+\frac{11}{60} \delta_{1}^{2}\right) \frac{\delta_{1} f}{\delta x}\right]+\left(I+\frac{1}{10} \delta_{1}^{2}\right)\left(I+\frac{5}{42} \delta_{2}^{2}\right) \frac{\delta_{2} \mu_{2} \mu_{1} g}{\delta y} \\
& \tilde{r}_{2}=\left(I+\frac{2}{7} \delta_{1}^{2}+\frac{1}{70} \delta_{1}^{4}\right)\left[\left(I+\frac{1}{10} \delta_{2}^{2}\right) \mu_{2} \frac{\partial w}{\partial t}+\left(I+\frac{11}{60} \delta_{2}^{2}\right) \frac{\delta_{2} g}{\delta y}\right]+\left(I+\frac{1}{10} \delta_{2}^{2}\right)\left(I+\frac{5}{42} \delta_{1}^{2}\right) \frac{\delta_{1} \mu_{1} \mu_{2} f}{\delta x}
\end{aligned}
$$

For later comparison with a second-order scheme, we also mention the $R B C_{2}$ scheme for which the eight scheme-coefficients $\bar{a}, \bar{b}, \bar{c}, a, b, c, a^{\mu}, a^{\delta}$ are null.

- Residuals for the $R B C_{2}$ scheme:

$$
\begin{gathered}
\tilde{r}=\frac{\partial w}{\partial t}+\frac{\delta_{1} \mu_{1} f}{\delta x}+\frac{\delta_{2} \mu_{2} g}{\delta y} \\
\tilde{r}_{1}=\mu_{1} \frac{\partial w}{\partial t}+\frac{\delta_{1} f}{\delta x}+\frac{\delta_{2} \mu_{2} \mu_{1} g}{\delta y} \\
\tilde{r}_{2}=\mu_{2} \frac{\partial w}{\partial t}+\frac{\delta_{2} g}{\delta y}+\frac{\delta_{1} \mu_{1} \mu_{2} f}{\delta x}
\end{gathered}
$$

Note this second-order scheme does not belong to the above class of $R B C_{2 p-1}$ schemes. In $R B C_{2}$, the residuals $\tilde{r}, \tilde{r}_{1}, \tilde{r}_{2}$ are three approximations of order 2 of the exact residual, so that the dispersive error (of order 2) dominates the dissipative error (of order 3) contrary to the situation for the $R B C_{2 p-1}$ schemes.

\section{High order time-approximation for the $R B C$ schemes}

\subsection{Current time-approximation}

Let us first introduce the time formulation in the one-dimensional case. For the hyperbolic system:

$$
\frac{\partial w}{\partial t}+\frac{\partial f}{\partial x}=0
$$

the general 5-point $R B C$ scheme reads:

$$
\left(I+\bar{b} \delta_{1}^{2}+\bar{c} \delta_{1}^{4}\right) \frac{\partial w}{\partial t}+\left(I+\bar{a} \delta_{1}^{2}\right) \frac{\delta_{1} \mu_{1} f}{\delta x}=\frac{1}{2} \delta_{1}\left\{\Phi_{1}\left[\left(I+a^{\mu} \delta_{1}^{2}\right) \mu_{1} \frac{\partial w}{\partial t}+\left(I+a^{\delta} \delta_{1}^{2}\right) \frac{\delta_{1} f}{\delta x}\right]\right\}
$$

A peculiarity of the $R B C$ schemes is the multiple occurrence of the time derivative in the scheme $(\partial w / \partial t$ occurs $d+1$ times in $d$-dimension). Besides, due to compactness, discrete spatial-operators are applied to each time-derivative. In previous applications of the $R B C$ schemes to unsteady problems (see $[17,4,5,18]$ for instance), the time formulation was based on the classical Gear method, that is all the terms of the scheme were taken at the new level $t=(n+1) \Delta t$ and the time derivative was approximated everywhere by

$$
\left(\frac{\partial w}{\partial t}\right)^{n+1}=\frac{1}{2 \Delta t}\left(3 w^{n+1}-4 w^{n}+w^{n-1}\right)+\mathcal{O}\left(\Delta t^{2}\right)
$$

This 3 time-level method is $A$-stable. However such a fully implicit treatment requires an iterative method. A dual-time stepping procedure was chosen in [4]. In dual time, it uses a first-order implicit scheme of Roe-Harten type with a large CFL number. At each dual-time iteration, this scheme is solved using a single iteration of an alternate-line symmetric Gauss-Seidel relaxation. This method is efficient for slow unsteady problems. For quick unsteady evolutions, it is costly and not accurate enough. Of course, the accuracy order could be increased by adding new time-levels in the above derivative approximation, which leads to the general implicit Backward-Differentiation Formulas $(i B D F)$, but $A$-stability cannot be preserved beyond order 2. Thus, we rather investigate the use of an explicit method of high accuracy. 


\subsection{Shifting the time-derivative in the numerical dissipation}

To construct an explicit time-stepping scheme, an attractive idea is to shift backward the time discretization of $\partial w / \partial t$ in the numerical dissipation. This idea has been proposed and developed for second-order residual-distribution schemes on an unstructured mesh by Ricchiuto and Abgrall in [19], using a Runge-Kutta method. To apply this approach to the $R B C$ scheme (26), we first rewrite it as

$$
D_{1} \frac{\partial w}{\partial t}=H_{0}+M_{1} \frac{\partial w}{\partial t}
$$

where $D_{1}$ and $M_{1}$ denote the linear operators:

$$
D_{1}=I+\bar{b} \delta_{1}^{2}+\bar{c} \delta_{1}^{4}, \quad M_{1}=\frac{1}{2} \delta_{1}\left[\left(\Phi_{1}\left(I+a^{\mu} \delta_{1}^{2}\right) \mu_{1} \cdot\right]\right.
$$

and $H_{0}$ is the steady flux balance:

$$
H_{0}=H_{0}(w)=-\left(I+\bar{a} \delta_{1}^{2}\right) \frac{\delta_{1} \mu_{1} f}{\delta x}+\frac{1}{2} \delta_{1}\left[\Phi_{1}\left(I+a^{\delta} \delta_{1}^{2}\right) \frac{\delta_{1} f}{\delta x}\right]
$$

The numerical dissipation of the $R B C$ scheme is entirely in the right-hand side of (27) through the second term of $H_{0}$ and the unsteady term $M_{1} \partial w / \partial t$. The Runge-Kutta method of order 2 with a shifted timederivative in the numerical dissipation can be written as $R K_{2} s d$ :

$$
\left\{\begin{array}{l}
D_{1} \frac{w^{(1)}-w^{n}}{\Delta t}=H_{0}^{n} \\
D_{1} \frac{w^{n+1}-w^{n}}{\Delta t}=\frac{1}{2}\left(H_{0}^{n}+H_{0}^{(1)}\right)+M_{1} \frac{w^{(1)}-w^{n}}{\Delta t}
\end{array}\right.
$$

Similarly, the Runge-Kutta method of order 3 with a shifted time-derivative in the numerical dissipation is $R K_{3} s d$ :

$$
\left\{\begin{array}{l}
D_{1} \frac{w^{(1)}-w^{n}}{\Delta t}=H_{0}^{n} \\
D_{1} \frac{w^{(2)}-w^{n}}{\Delta t / 2}=\frac{1}{2}\left(H_{0}^{n}+H_{0}^{(1)}\right)+M_{1} \frac{w^{(1)}-w^{n}}{\Delta t} \\
D_{1} \frac{w^{n+1}-w^{n}}{\Delta t}=\frac{1}{3}\left[\frac{1}{2}\left(H_{0}^{n}+H_{0}^{(1)}\right)+2 H_{0}^{(2)}\right]+M_{1} \frac{w^{(2)}-w^{n}}{\Delta t / 2}
\end{array}\right.
$$

where $H_{0}^{n}=H_{0}\left(w^{n}\right), H_{0}^{(p)}=H_{0}\left(w^{(p)}\right), p=1,2$. Note that the predictors $w^{(1)}$ and $w^{(2)}$ are defined at $t=(n+1) \Delta t$ and $\left(n+\frac{1}{2}\right) \Delta t$, respectively.

It has been proved in [19] that $R K_{2} s d$ with a second-order spatial discretization is overall of order 2 and similarly $R K_{3} s d$ with a third-order spatial discretization is overall of order 3 . This can be understood by noting that $M_{1} \approx \frac{\delta x}{2} \frac{\partial}{\partial x}\left(\Phi_{1}.\right)=\mathcal{O}(\delta x)$.

Used with the present $R B C$ scheme, these $R K_{q} s d$ methods only requires the solution of $q$ simple linear systems (tridiagonal for $R B C_{3}$ and $R B C_{5}$ or pentadiagonal for $R B C_{7}$ ) for each component of $w$. For a two-dimensional problem, $D_{1}$ in (28) or (29) is replaced by $D_{1} D_{2}$, which comes to solve the linear systems on each mesh lines of the 2-D mesh.

Unfortunately, our numerical experiments show that this approach (as well as several variants of it) fails when the time-accuracy order is greater than 2, for stability reasons -see Section 4-.

\subsection{Direct extraction of the time-derivative}

We now consider a direct extraction of the continuous time derivative $\partial w / \partial t=\left[(\partial w / \partial t)_{j}\right]$ from the linear system (27) rewritten as:

$$
\Lambda_{1} \frac{\partial w}{\partial t}=H_{0}
$$

where

$$
\Lambda_{1}=D_{1}-M_{1}
$$


and $D_{1}, M_{1}$ and $H_{0}$ are still defined as above. To find the time derivative, we have now to solve a blocktridiagonal system for $R B C_{3}$ and $R B C_{5}$ and a block-pentadiagonal system for $R B C_{7}$. The block structure is due to the matrix $\Phi_{1}$ in the numerical dissipation, so that the blocks have the same size as the Jacobian matrix $A=d f / d w$. System (30) is well-conditioned and can be easily solved by a direct method.

Let us extend this direct approach to the two-dimensional system (1). We have to extract the time derivative from the linear algebraic system:

$$
\Lambda \frac{\partial w}{\partial t}=H_{0}
$$

where $\Lambda$ denotes the linear operator:

$$
\Lambda=D_{1} D_{2}-D_{2} M_{1}-D_{1} M_{2}
$$

with

$$
\begin{array}{ll}
D_{1}=I+\bar{b} \delta_{1}^{2}+\bar{c} \delta_{1}^{4}, & M_{1}=\frac{1}{2} \delta_{1}\left[\left(\Phi_{1}\left(I+a^{\mu} \delta_{1}^{2}\right) \mu_{1} \cdot\right]\right. \\
D_{2}=I+\bar{b} \delta_{2}^{2}+\bar{c} \delta_{2}^{4}, & M_{2}=\frac{1}{2} \delta_{2}\left[\left(\Phi_{2}\left(I+a^{\mu} \delta_{2}^{2}\right) \mu_{2} \cdot\right]\right.
\end{array}
$$

and $H_{0}$ is the steady flux balance:

$$
H_{0}=-\tilde{r}^{0}+\frac{1}{2}\left[\delta_{1}\left(\Phi_{1} \tilde{r}_{1}^{0}\right)+\delta_{2}\left(\Phi_{2} \tilde{r}_{2}^{0}\right)\right]
$$

$\tilde{r}^{0}, \tilde{r}_{1}^{0}$ and $\tilde{r}_{2}^{0}$ being the general residuals defined by (5) and (9) in which $\partial w / \partial t=0$.

However, the algebraic structure of $\Lambda$ is more complicated than in one dimension. To simplify the problem, we attempt a dimensional factorization of $\Lambda$ :

$$
\Lambda=\Lambda_{1} \Lambda_{2}-\Lambda_{c}
$$

where

$$
\Lambda_{1}=D_{1}-M_{1}, \quad \Lambda_{2}=D_{2}-M_{2}, \quad \Lambda_{c}=M_{1} M_{2}
$$

As $D_{1}$ and $D_{2}$, the one-dimensional operators $\Lambda_{1}$ and $\Lambda_{2}$ are locally $\mathcal{O}(1)$, but since $M_{1}=\mathcal{O}(\delta x)$ and $M_{2}=\mathcal{O}(\delta y)$, the corrective operator $\Lambda_{c}$ is locally $\mathcal{O}(\delta x \delta y)=\mathcal{O}\left(h^{2}\right)$. Thus, contrary to common practice in the eighties when second order methods were used, we cannot neglect $\Lambda_{c}$ and achieve a standard approximatefactorization.

Let us rewrite the linear system (31) in the form:

$$
\Lambda_{1} \Lambda_{2} \frac{\partial w}{\partial t}=H^{0}+\Lambda_{c} \frac{\partial w}{\partial t}
$$

It can be solved approximately in a few iterations as:

$$
\Lambda_{1} \Lambda_{2}{\frac{\partial w^{(m+1)}}{\partial t}}^{\left(m+H_{c}\right.}{\frac{\partial w^{(m)}}{\partial t}}^{0}, \quad m=0,1, \ldots, m_{f}
$$

starting from

$$
{\frac{\partial w^{(0)}}{\partial t}}^{(0)}=0
$$

Eq. (34) can be split into:

$$
\left\{\begin{array}{l}
\Lambda_{1} \frac{\partial \tilde{w}}{\partial t}=H^{0}+\Lambda_{c} \frac{\partial w}{\partial t}^{(m)} \\
\Lambda_{2} \frac{\partial w}{\partial t}^{(m+1)}=\frac{\partial \tilde{w}}{\partial t}
\end{array}\right.
$$


Thus, we have only to solve simple linear systems (block-tridiagonal for $R B C_{3}$ and $R B C_{5}$ or blockpentadiagonal for $R B C_{7}$ ) in each space direction. Let us now determine the required number of iterations $m_{f}$. By substracting (33) to (34), we get:

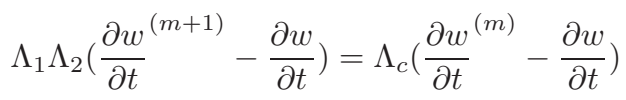

and therefore

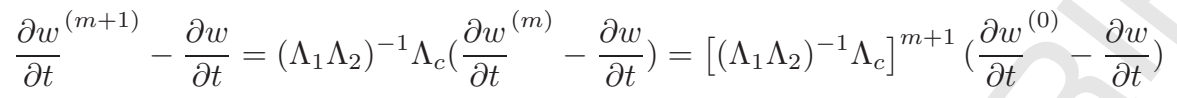

Taking into account (35), we obtain the local error of the iterative procedure after $m_{f}$ iterations:

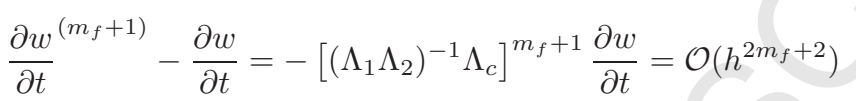

We can conclude that for $m_{f}=0$ (standard approximate factorization), the space accuracy is limited to 2nd order. For $m_{f}=1$ (one iteration), it is limited to 4 th order, which is quite sufficient for $R B C_{3}$. For $m_{f}=2$ (resp. $m_{f}=3$ ), it is limited to 6th order (resp. 8th order), which is quite sufficient for $R B C_{5}$ (resp. $R B C_{7}$ ). So, very few iterations (a number known in advance for a given space-accuracy) over 1-D linear systems garantee the high accuracy in space. It is worth noting than MacCormack [20] studied in 2001 the approximate factorization technique for second-order methods applied to fluid flows. He noticed that factorization errors cause a reduction in convergence speed and the necessity of using time steps much smaller than that required to follow the time evolution of unsteady flows, particularly viscous flows or those converging to steady-state solutions. So he proposed a modified approximate factorization involving an iterative method having some similarity with the present approach. Although the iterations in (36) are intended to garantee the high space-accuracy, we have checked that they also improve the stability of the method, especially the first iteration.

\subsection{Choice of a high order time-integration}

After the direct extraction of the time derivative at time $t$, we advance the numerical solution by solving an ordinary differential equation (ODE) of the form:

$$
\frac{\partial w}{\partial t}=H(w)
$$

For the present $R B C$ schemes, the main cost per time step comes from the calculation of $H=\Lambda^{-1} H_{0}$ as the solution of the problem (36). So we are interested in ODE methods requiring few evaluations of $H$ per time-step. We first consider the class of linear multistep methods. A possible candidate is the AdamsBashforth method of order q $\left(A B_{q}\right)$ since it only requires a single evaluation of $\mathrm{H}$ per time step, for any order q. Adams-Bashforth methods of third and fourth accuracy-order are recalled below.

- $A B_{3}$ method:

$$
w^{n+1}=w^{n}+\frac{\Delta t}{12}\left(23 H^{n}-16 H^{n-1}+5 H^{n-2}\right)
$$

- $A B_{4}$ method:

$$
w^{n+1}=w^{n}+\frac{\Delta t}{24}\left(55 H^{n}-59 H^{n-1}+37 H^{n-2}-9 H^{n-3}\right)
$$

where $w^{n}=\left(w_{j}^{n}\right)$ denotes the numerical solution at time level $t^{n}=n \Delta t$ and $H^{n}=H\left(w^{n}\right)$. The $A B_{q}$ method involves $q+1$ time levels.

Still in the class of linear multistep methods, Hundsdorfer, Ruuth and Spiteri [21] have proved that the explicit -or extrapolated- Backward Differentiation Formulas $(e B D F)$ of order 3 and 4 have better boundedness and TVB properties than $A B$ methods of the same order, which allows the use of greater time-steps. 
So we also consider:

- eBDF method:

$$
w^{n+1}=\frac{1}{11}\left(18 w^{n}-9 w^{n-1}+2 w^{n-2}\right)+\frac{6 \Delta t}{11}\left(3 H^{n}-3 H^{n-1}+H^{n-2}\right)
$$

- eBDF 4 method:

$$
w^{n+1}=\frac{1}{25}\left(48 w^{n}-36 w^{n-1}+16 w^{n-2}-3 w^{n-3}\right)+\frac{\Delta t}{25}\left(48 H^{n}-72 H^{n-1}+48 H^{n-2}-12 H^{n-3}\right)
$$

Note that $e B D F_{q}$ involves the same number of time level as $A B_{q}$. However in $e B D F_{q}$, the time levels apply not only to $H$ but also to $w$, which increases the memory requirements.

An improvement of the Adams-Bashforth method is to combine it with its implicit version, i.e. the AdamsMoulton method $(A M)$, in order to get an explicit predictor-corrector method called Adams-BashforthMoulton $(A B M)$. More precisely, in the $A B M_{q}$ method, the predictor step is $A B_{q-1}$ and the corrector step is a modified $A M_{q}$ in which the flux balance $H^{n+1}$ is computed from the state $\tilde{w}^{n+1}$ obtained at the predictor step -see e.g. [22]-. At order 3 and 4, this method can be written as:

- $A B M_{3}$ method:

$$
\left\{\begin{array}{l}
\tilde{w}^{n+1}=w^{n}+\frac{\Delta t}{2}\left(3 H^{n}-H^{n-1}\right) \\
w^{n+1}=w^{n}+\frac{\Delta t}{12}\left(5 \tilde{H}^{n+1}+8 H^{n}-H^{n-1}\right)
\end{array}\right.
$$

- $A B M_{4}$ method:

$$
\left\{\begin{array}{l}
\tilde{w}^{n+1}=w^{n}+\frac{\Delta t}{12}\left(23 H^{n}-16 H^{n-1}+5 H^{n-2}\right) \\
w^{n+1}=w^{n}+\frac{\Delta t}{24}\left(9 \tilde{H}^{n+1}+19 H^{n}-5 H^{n-1}+H^{n-2}\right)
\end{array}\right.
$$

where $\tilde{H}^{n+1}=H\left(\tilde{w}^{n+1}\right)$. Note that $A B M_{q}$ requires two evaluations of $H$ per time-step $\left(H^{n}\right.$ and $\left.\tilde{H}^{n+1}\right)$ for any order q, but uses one time-level less than $A B_{q}$ or $e B D F_{q}$.

Finally we consider the most used methods in high-accurate flow simulations, that is the Runge-Kutta methods $\left(R K_{q}\right)$. We recall below the $R K_{2}$ method (coinciding with $A B M_{2}$ ), the $R K_{3}$ method of Shu and Osher and a classical $R K_{4}$ method.

- $R K_{2}=A B M_{2}$ method:

$$
\left\{\begin{array}{l}
\tilde{w}^{(1)}=w^{n}+\Delta t H^{n} \\
w^{n+1}=w^{n}+\frac{\Delta t}{2}\left(H^{n}+\tilde{H}^{(1)}\right)
\end{array}\right.
$$

- $R K_{3}$ method:

- $R K_{4}$ method:

$$
\left\{\begin{array}{l}
\tilde{w}^{(1)}=w^{n}+\Delta t H^{n} \\
\tilde{w}^{(2)}=w^{n}+\frac{\Delta t}{4}\left(H^{n}+\tilde{H}^{(1)}\right) \\
w^{n+1}=w^{n}+\frac{\Delta t}{6}\left(H^{n}+\tilde{H}^{(1)}+4 \tilde{H}^{(2)}\right)
\end{array}\right.
$$

$$
\left\{\begin{array}{l}
\tilde{w}^{(1)}=w^{n}+\frac{\Delta t}{2} H^{n} \\
\tilde{w}^{(2)}=w^{n}+\frac{\Delta t}{2} \tilde{H}^{(1)} \\
\tilde{w}^{(3)}=w^{n}+\Delta t \tilde{H}^{(2)} \\
w^{n+1}=w^{n}+\frac{\Delta t}{6}\left(H^{n}+2 \tilde{H}^{(1)}+2 \tilde{H}^{(2)}+\tilde{H}^{(3)}\right)
\end{array}\right.
$$

where $\tilde{H}^{(r)}=H\left(\tilde{w}^{(r)}\right)$. The drawback of the $R K_{q}$ method is to compute $H$ as many times as the timeaccuracy order $q$, which can be rather costly as $q$ increases. In return, they are known to have greater 
stability limits than linear multistep methods.

The above four ODE-methods of order $4\left(A B_{4}, e B D F_{4}, A B M_{4}, R K_{4}\right)$ applied to the $R B C_{5}$ scheme have been compared for a long-time integration of a 2-D Euler problem: the vortex advection described in Section 4.3. The multistep methods are started by computing the first time-iterations with $R K_{4}$. The four ODEmethods use (36) with $m_{f}=2$ to compute the flux-balance $H$ a number of times between 1 and 4 . They also have quite different time-step limits for stability. Using the maximal time-step for each method, the CPU time for reaching the physical time $\mathrm{t}=100$ is given in Table 1 on a single processor Intel-X5680 (year 2010) for a non-optimized code written for all the RBC schemes of the general form (4)-(5)-(9). Note that concerning the accuracy at $\mathrm{t}=100$, the results given by the four ODE-methods are very close (practically the same $L_{2}$-error). The $A B M_{4}$ time-integration is the cheapest: its CPU-time is $25 \%$ lower than $e B D F_{4}$ and $R K_{4}$ and more than 3 times lower than $A B_{4}$. On the other hand, $R K_{4}$ is the least memory-consuming. Noting that the advantage in CPU time of $A B M$ over $R K$ will grow up with more complicated flux-balance $H$ (like that of the compressible Navier-Stokes equations), we choose here to retain the quickest method, i.e. the $A B M$ time-integration.

\begin{tabular}{ccccc}
\hline & $R B C_{5}-A B_{4}$ & $R B C_{5}-e B D F_{4}$ & $R B C_{5}-A B M_{4}$ & $R B C_{5}-R K_{4}$ \\
\hline$H$ computations per time step & 1 & 1 & 2 & 4 \\
$\Delta t$ (maximal value) & 0.0032 & 0.008 & 0.020 & 0.032 \\
CPU time (sec.) for $t=100$ & 286.5 & 114.3 & 91.4 & 114.5 \\
\hline
\end{tabular}

Table 1: Computational cost on Intel-X5680 of several time-integrations for a 2-D Euler problem (vortex advection during a long time on a 50x50 mesh with periodicity conditions)

\section{Numerical validations for 2-D problems}

The new time-formulation of the $R B C$ schemes has been applied to several two-dimensional problems governed by a linear hyperbolic equation with constant or variable coefficients and the compressible Euler equations. We focuss the validation of the time-formulation on the $R B C_{2}, R B C_{3}$ and $R B C_{5}$ spatial schemes which are especially cheap since they only require the solution of block-tridiagonal systems. Let us point out that all the validations have been made without any correction of the formulas given above. In particular, there is no limiter, no entropy correction, no filtering or other additive.

\subsection{Diagonal advection of a Gaussian}

Consider the diagonal advection of an axisymmetric Gaussian shape in a square domain:

$$
\left\{\begin{array}{l}
w_{t}+w_{x}+w_{y}=0 \\
w(x, y, 0)=\exp \left(-75 r^{2}\right), \quad(x, y) \in[-1,1]^{2}
\end{array}\right.
$$

with $r^{2}=\left(x-x_{c}\right)^{2}+\left(y-y_{c}\right)^{2}$ and Dirichlet boundary conditions on left and bottom sides. The Gaussian is initially centered at $x_{c}=y_{c}=-0.5$ and moves diagonally at speed $\sqrt{ } 2$. This problem is solved until time We first consider the approach of shifting the time-derivative in the numerical dissipation presented in Section 3.2. A solution is computed by the $R B C_{2}-R K_{2} s d$ scheme on a $80 \times 80$ mesh with $\Delta t=1 / 400$ (maximal time-step for stability). Fig.1 shows the $w$-isolines at times $t=0, t=0.5$ and $t=1$, together with the Gaussian profiles at the initial and final times. On the same mesh, we have not been able to run the $R B C_{3}-R K_{3} s d$ scheme, even with very small time-steps. Using $R B C_{3}-R K_{2} s d$, we have obtained the solution plotted on Fig. 2 with $\Delta t=1 / 3200$.

Now we test the direct extraction of the time derivative. Solutions computed on a $80 \times 80$ mesh with $\Delta t=$ 
$1 / 100$ by the scheme $R B C_{2}-A B M_{2}$ without iteration $\left(m_{f}=0\right)$ and with one iteration $\left(m_{f}=1\right)$ are shown on Fig.3-4. Although second-order accuracy is ensured without iteration, iterating once gives a better solution which is really that of the original formulation. As a matter of fact, if the term $\Lambda_{c}=M_{1} M_{2}$ is not corrected, the scheme is no longer residual-based. The third-order solution given by $R B C_{3}-A B M_{3}\left(m_{f}=1\right)$ on a the same mesh with $\Delta t=1 / 180$ is shown on Fig.5. For the $R B C_{3}$ spatial-scheme, the direct extraction technique is 9 times faster in CPU-time and 11 times more accurate in $L_{2}$ norm than the simplified technique using a time shifting in the dissipation $\left(R B C_{3}-R K_{2} s d\right)$. The direct extraction using the $R B C_{5}-A B M_{4}$ $\left(m_{f}=2\right)$ on the same mesh with $\Delta t=1 / 200$ produces the very accurate solution shown on Fig.6. Note that the solution given by $R B C_{5}-A B M_{4}$ with $m_{f}=1$ is very close to that of Fig.6 at the present scale. Let us point out that if the factorization error is not corrected at all, i.e. no iteration is done $\left(m_{f}=0\right)$, the schemes $R B C_{3}-A B M_{3}$ and $R B C_{5}-A B M_{4}$ cannot be run, even with very small time-steps. With one iteration, $R B C_{5}$ becomes theoretically accurate at order 4 only. To determine the actual levels of accuracy, we present on the left side of Fig. 7 the $L_{2}$-errors with respect to the $C^{\infty}$ exact-solution, versus $h=\delta x=\delta y$ on a series of meshes $(40 \mathrm{x} 40,80 \mathrm{x} 80,160 \mathrm{x} 160,320 \mathrm{x} 320)$. These errors have been computed at time $t=1$ in the square $[0,1]^{2}$ for $R B C_{2}-A B M_{2}, R B C_{3}-A B M_{3}$ with $m_{f}=1$ and $R B C_{5}-A B M_{4}$ with $m_{f}=1$ and $m_{f}=2$. The slopes in the log-log scale are in perfect agreement with the theory: as predicted in Section 3 , $m_{f}=2$ iterations are enough to ensure negligible space-factorization error, while setting $m_{f}=1$ limits the order to four. The right side of Fig.7 shows the effect of the time-integration order on $R B C_{5}$ with $m_{f}=2$ : replacing $A B M_{4}$ by $A B M_{3}$ reduces the global error-order from 4.97 to 3.39 .
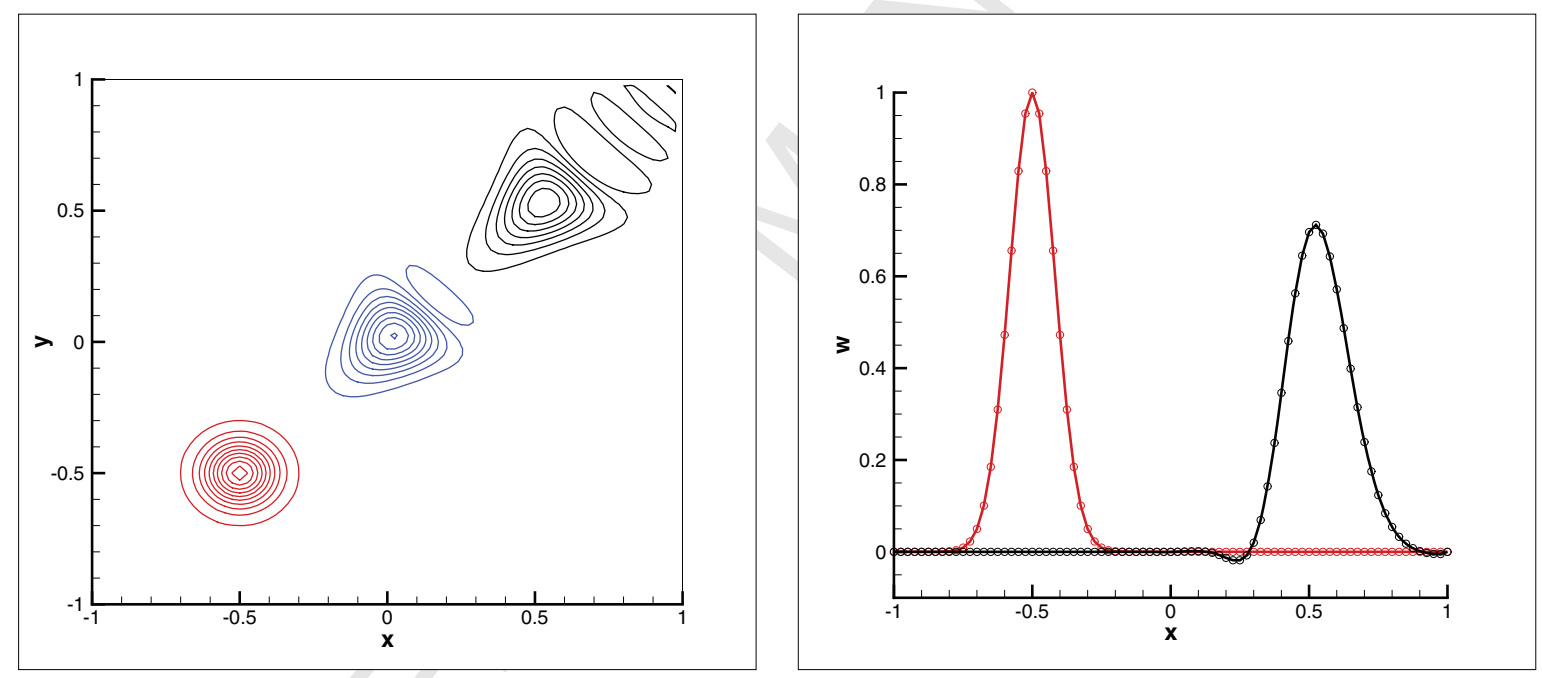

Figure 1: Diagonal advection of a Gaussian by $R B C_{2}-R K_{2} s d$ scheme on a $80 x 80$ mesh. Left: isolines (from $w=-0.05$ to $w=1.05, \Delta w=0.1)$ at $t=0,0.5,1$. Right: $\mathrm{x}$-profiles with mesh points at $t=0(y=-0.5)$ and $t=1(y=0.5)$. 

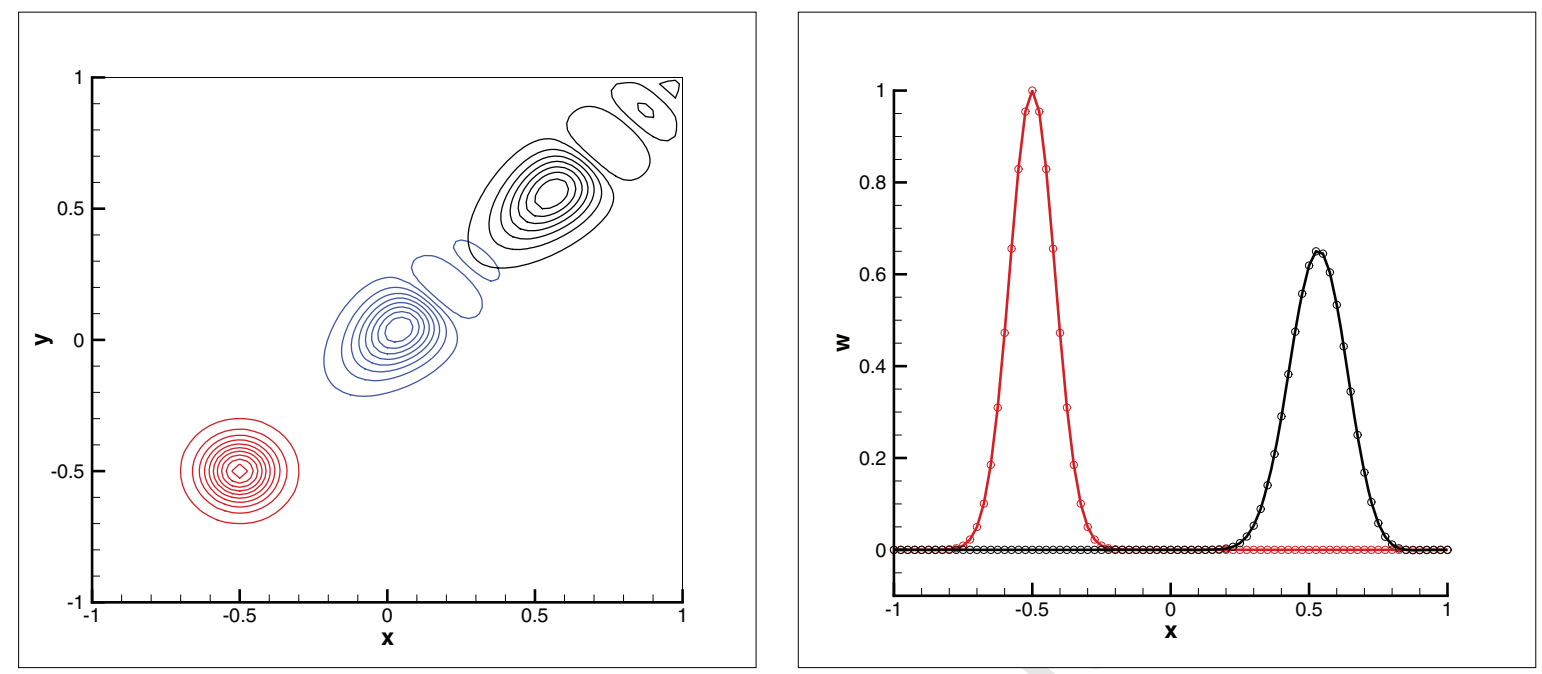

Figure 2: Diagonal advection of a Gaussian by $R B C_{3}-R K_{2} s d$ scheme on a $80 \times 80$ mesh. Left: isolines (from $w=-0.05$ to $w=1.05, \Delta w=0.1)$ at $t=0,0.5,1$. Right: $\mathrm{x}$-profiles with mesh points at $t=0(y=-0.5)$ and $t=1(y=0.5)$.
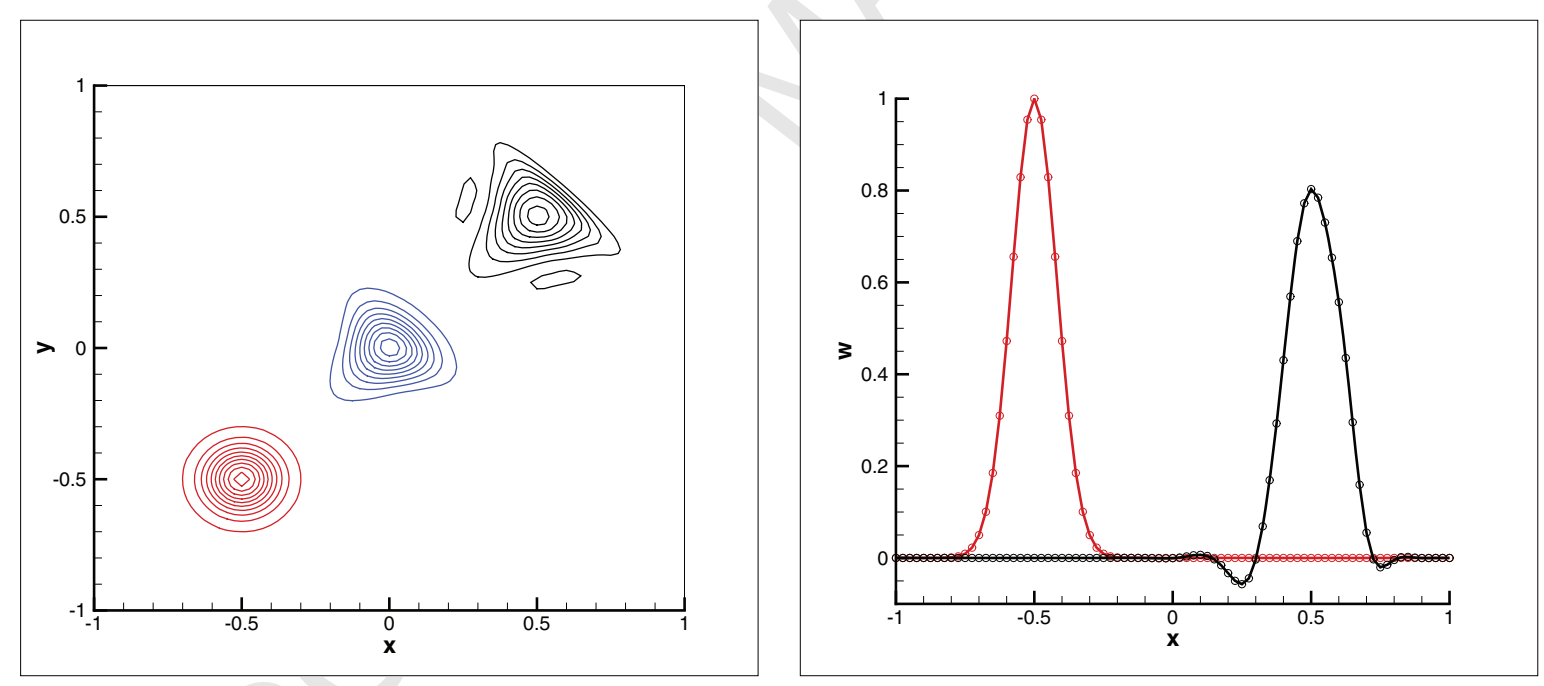

Figure 3: Diagonal advection of a Gaussian by $R B C_{2}-A B M_{2}\left(m_{f}=0\right)$ scheme on a $80 x 80$ mesh. Left: isolines (from $w=-0.05$ to $w=1.05, \Delta w=0.1)$ at $t=0,0.5,1$. Right: x-profiles with mesh points at $t=0(y=-0.5)$ and $t=1(y=0.5)$. 

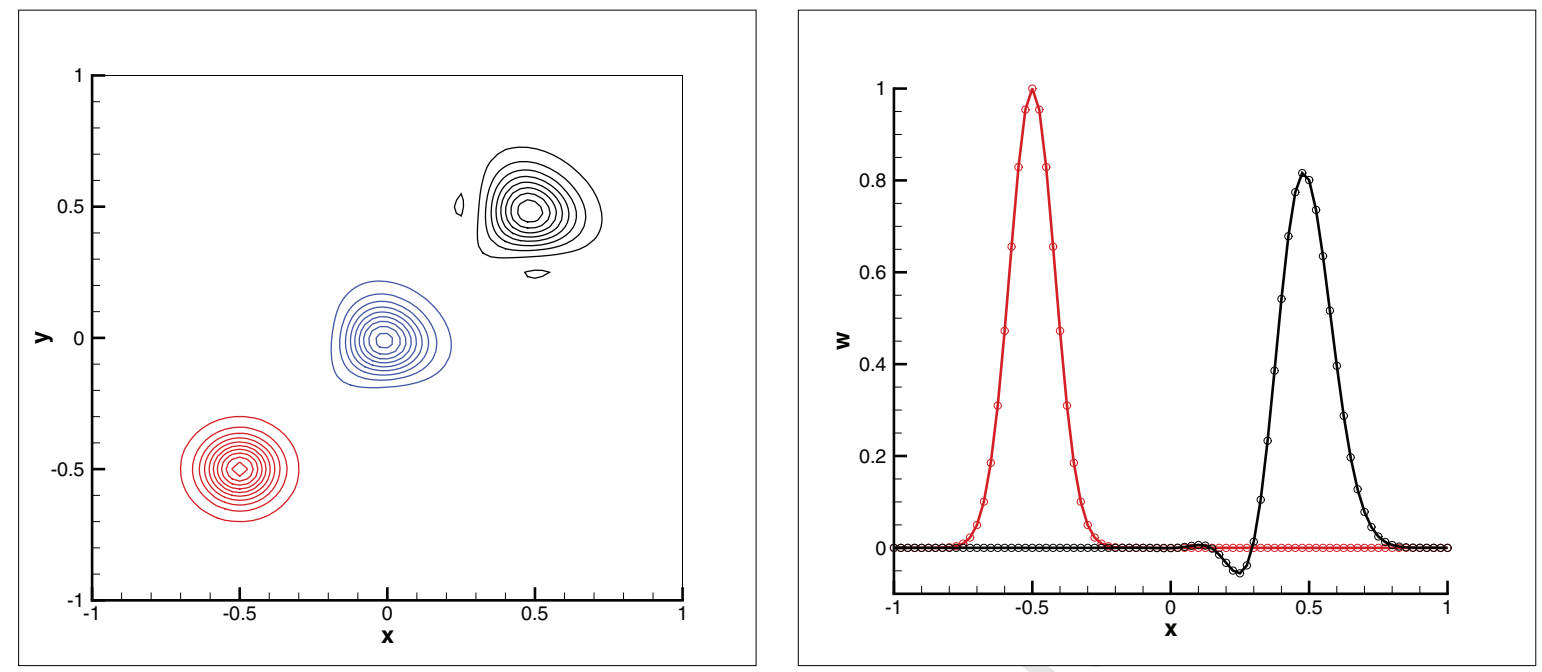

Figure 4: Diagonal advection of a Gaussian by $R B C_{2}-A B M_{2}\left(m_{f}=1\right)$ scheme on a $80 x 80$ mesh. Left: isolines (from $w=-0.05$ to $w=1.05, \Delta w=0.1)$ at $t=0,0.5,1$. Right: $\mathrm{x}$-profiles with mesh points at $t=0(y=-0.5)$ and $t=1(y=0.5)$.
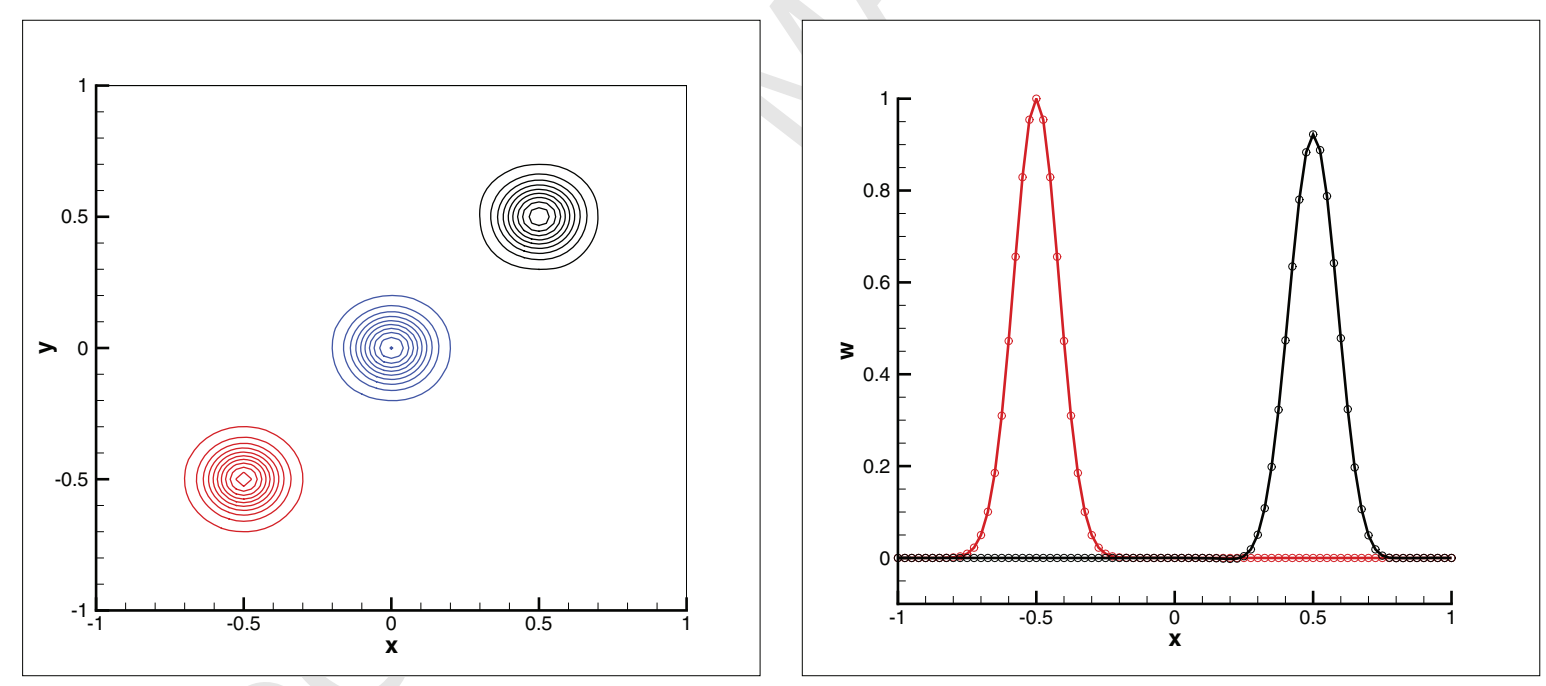

Figure 5: Diagonal advection of a Gaussian by $R B C_{3}-A B M_{3}\left(m_{f}=1\right)$ scheme on a $80 x 80$ mesh. Left: isolines (from $w=-0.05$ to $w=1.05, \Delta w=0.1)$ at $t=0,0.5,1$. Right: x-profiles with mesh points at $t=0(y=-0.5)$ and $t=1(y=0.5)$. 

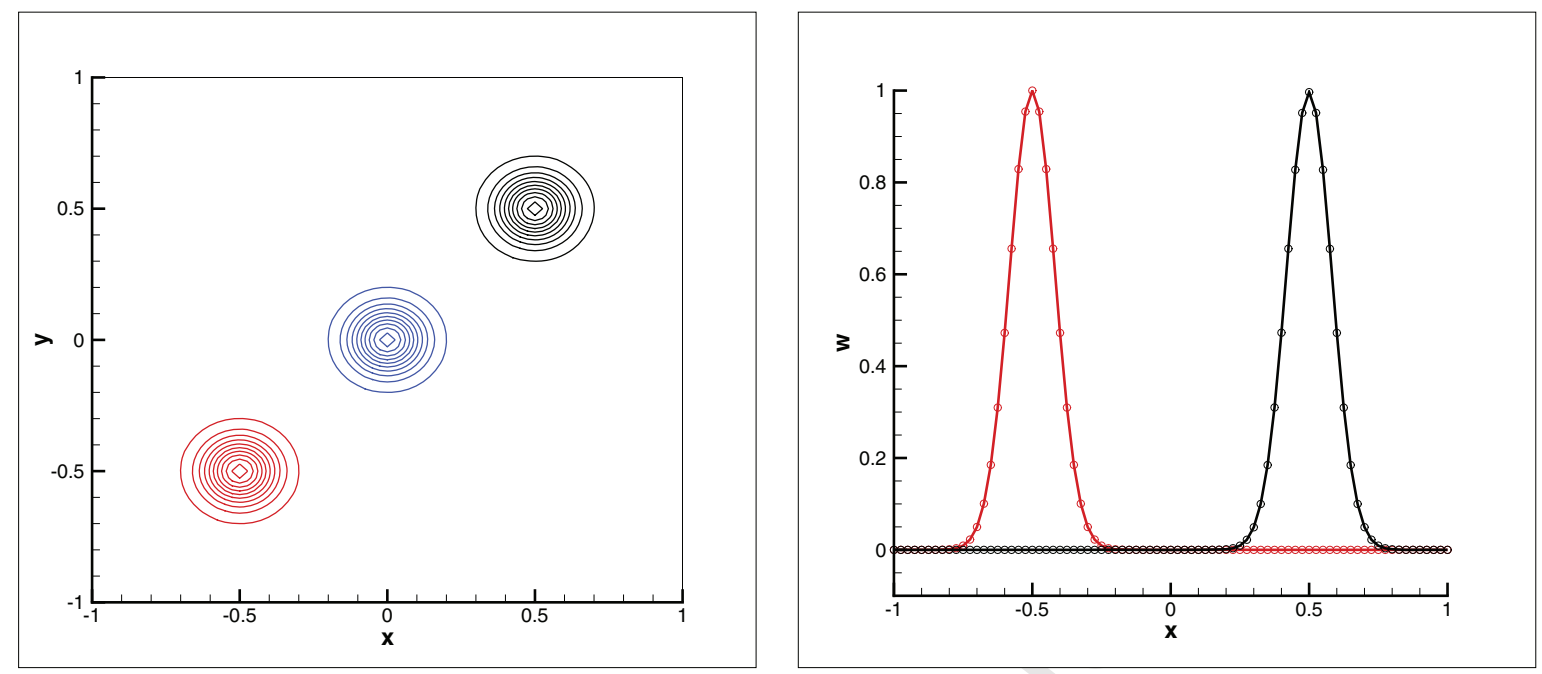

Figure 6: Diagonal advection of a Gaussian by $R B C_{5}-A B M_{4}\left(m_{f}=2\right)$ scheme on a $80 \times 80$ mesh. Left: isolines (from $w=-0.05$ to $w=1.05, \Delta w=0.1)$ at $t=0,0.5,1$. Right: $\mathrm{x}$-profiles with mesh points at $t=0(y=-0.5)$ and $t=1(y=0.5)$.
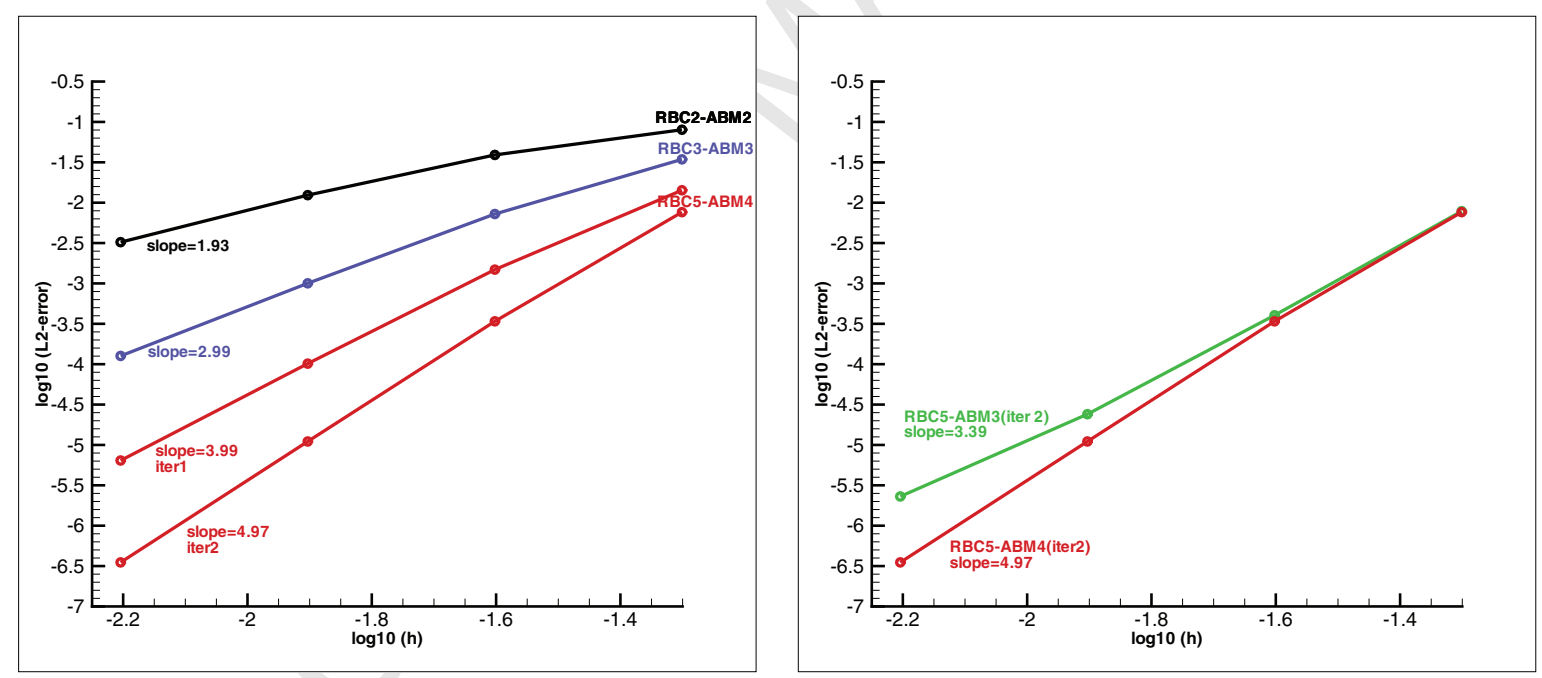

Figure 7: $L_{2}$ errors for the diagonal advection of a Gaussian on a series of meshes $(40 \mathrm{x} 40,80 \mathrm{x} 80,160 \mathrm{x} 160,320 \mathrm{x} 320)$. Left: $R B C_{2}-A B M_{2}\left(m_{f}=1\right), R B C_{3}-A B M_{3}\left(m_{f}=1\right)$ and $R B C_{5}-A B M_{4}\left(m_{f}=1,2\right)$. Right: $R B C_{5}-A B M_{3}\left(m_{f}=\right.$ $2)$ and $R B C_{5}-A B M_{4}\left(m_{f}=2\right)$.

\subsection{Rotation of a hump}

Consider the rotation of an axisymmetric hump in a square domain:

$$
\left\{\begin{array}{l}
w_{t}-2 \pi y w_{x}+2 \pi x w_{y}=0 \\
w(x, y, 0)=w^{0}(x, y),(x, y) \in[-1,1]^{2}
\end{array}\right.
$$


where

$$
w^{0}(x, y)= \begin{cases}\cos ^{2}(2 \pi r) & \text { for } r \leq 0.25 \\ 0 & \text { for } r>0.25\end{cases}
$$

with $r^{2}=\left(x-x_{c}\right)^{2}+\left(y-y_{c}\right)^{2}$ and $w=0$ as boundary condition. The hump is initially centered at $x_{c}=-0.5, y_{c}=0$. It turns counterclockwise making one revolution during a time unit.

Calculations are made on a $80 \times 80$ regular-mesh during the first revolution. The hump is initially discretized over 20 cells in $\mathrm{x}$ and $\mathrm{y}$-direction. For the shifting of the time-derivative in dissipation or its direct extraction and for the various accuracy-orders, the numerical results lead to conclusions quite similar to those obtained for the problem of diagonal advection. Here we just present two numerical results regarding the direct extraction approach. Fig.8 shows the solution given by the $R B C_{2}-A B M_{2}\left(m_{f}=1\right)$ scheme which has significant errors at time $t=1$. In contrast, the solution obtained with the $R B C_{5}-A B M_{4}\left(m_{f}=2\right)$ scheme in the same conditions seems perfect as shown on Fig.9.
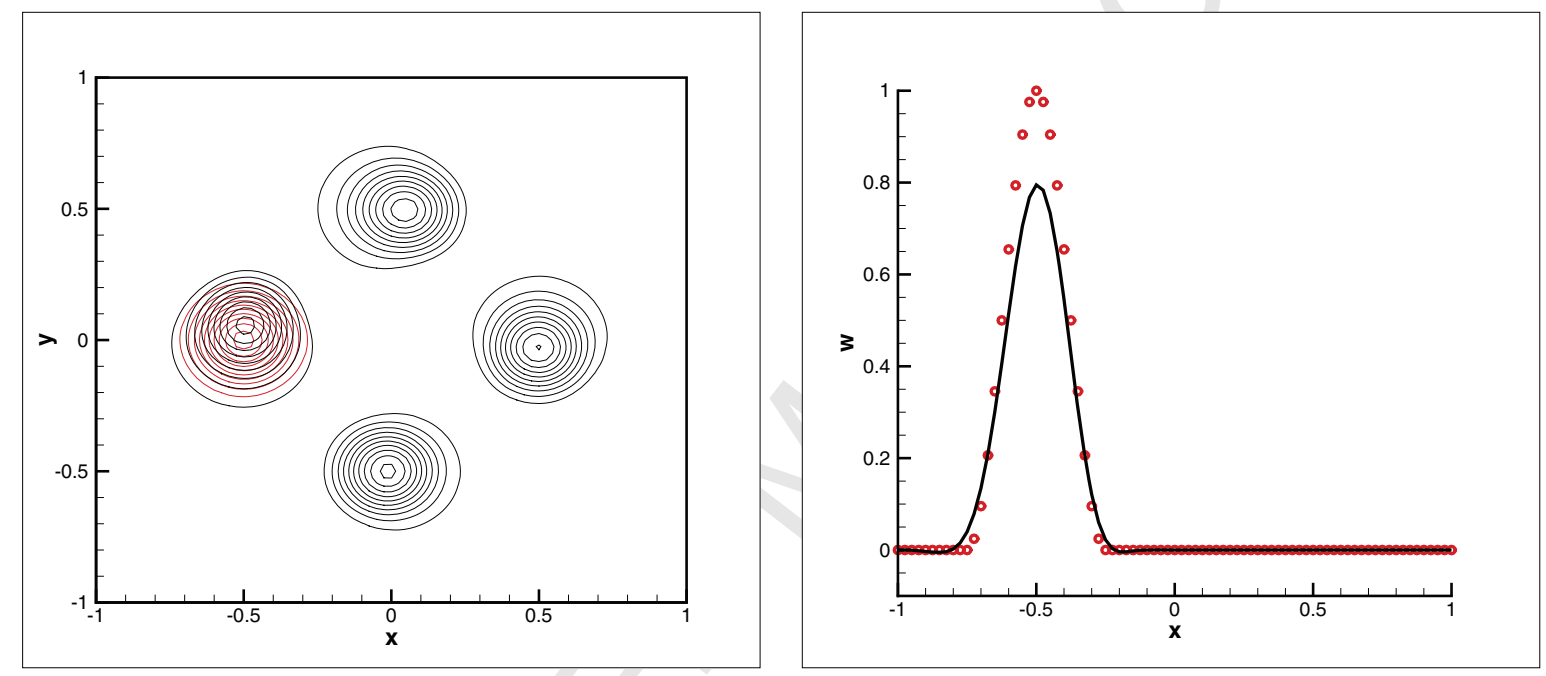

Figure 8: Rotation of a hump by $R B C_{2}-A B M_{2}\left(m_{f}=1\right)$ scheme on a $80 \times 80$ mesh. Left: isolines (from $w=-0.05$ to $w=1.05, \Delta w=0.1$ ) at $t=0,0.25,0.5,0.75,1$. Right: $\mathrm{x}$-profile on the axis $y=0$ at $t=0$ (symbols) and at $t=1$ (line). 

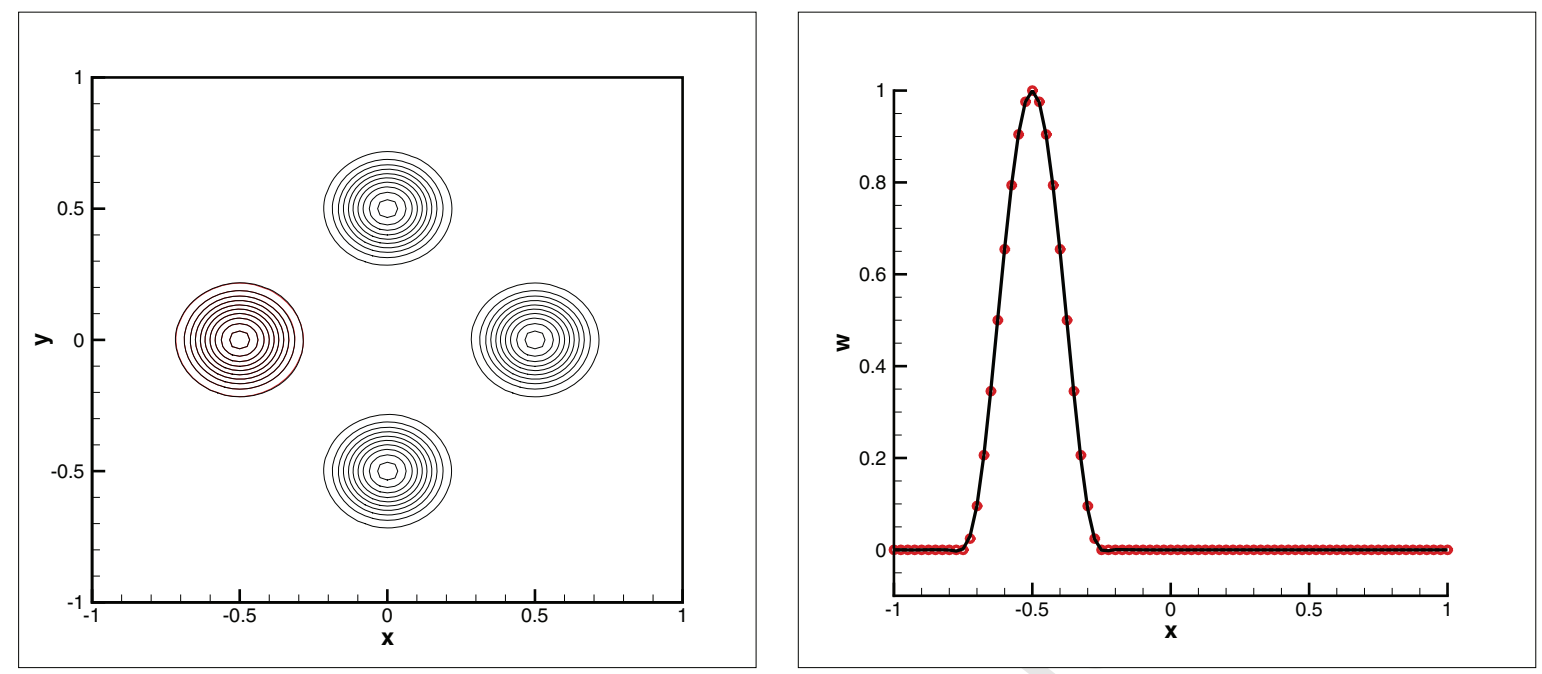

Figure 9: Rotation of a hump by $R B C_{5}-A B M_{4}\left(m_{f}=2\right)$ scheme on a $80 \times 80$ mesh. Left: isolines (from $w=-0.05$ to $w=1.05, \Delta w=0.1$ ) at $t=0,0.25,0.5,0.75,1$. Right: $\mathrm{x}$-profile on the axis $y=0$ at $t=0$ (symbols) and at $t=1$ (line).

\subsection{Advection of a vortex during a long time}

Consider now the Euler equations and an homentropic vortex initially located at the origin $(x=y=0)$ and advected in the $\mathrm{x}$-direction. The initial velocity components $u, v$ and the absolute temperature $T$ are defined in non-dimensional form as:

$$
\begin{gathered}
u=u_{0}-\frac{\Gamma}{2 \pi} y \exp \left(\frac{1-r^{2}}{2}\right) \quad v=\frac{\Gamma}{2 \pi} x \exp \left(\frac{1-r^{2}}{2}\right) \\
T=1-\frac{(\gamma-1) \Gamma^{2}}{8 \gamma \pi^{2}} \exp \left(1-r^{2}\right)
\end{gathered}
$$

with $r^{2}=x^{2}+y^{2}$, the advection speed $u_{0}=0.5$ and the vortex strength $\Gamma=5$. The thermodynamic equation of state is the ideal law $p=\rho T$, with constant specific heats of ratio $\gamma=1.4$. The uniformity of entropy gives $\rho=T^{1 / \gamma-1}$. The computational domain is $[-5,5]^{2}$. Periodic boundary conditions are applied in both directions. Calculations are made until time $t=100$, after which the vortex has traveled a distance of 50. All calculations use a 50x50 regular-mesh so that the vortex is discretized over about 20 cells in $\mathrm{x}$ and y-direction.

The simplified technique using a time-shifting in dissipation behaves similarly as for scalar problems. For the $R B C_{2}-R K_{2} s d$ scheme with $\Delta t=1 / 100$ (maximal time-step), Fig.10 shows the $w$-isobar lines at times $t=0$ and $t=100$, together with a pressure cut on $y=0$ at the initial and final times. The $R B C_{3}-R K_{2} s d$ scheme works with $\Delta t=1 / 400$ at most and produces the results shown on Fig.11. The $R B C_{3}-R K_{3} s d$ scheme is unstable.

Concerning the direct extraction of the time derivative, $R B C_{2}-A B M_{2}\left(m_{f}=1\right)$ can be run with $\Delta t=1 / 20$, $R B C_{3}-A B M_{3}\left(m_{f}=1\right)$ with $\Delta t=1 / 40$ and $R B C_{5}-A B M_{4}\left(m_{f}=1\right)$ with $\Delta t=1 / 50$. In order to compare these three schemes for the same time-step, we present their solutions at time $t=100$ with $\Delta t=1 / 50$. The solution given by the second-order scheme is shown on Fig.12. The second-order phase error is mainly responsible for the poor result. A better solution can be obtained by the third-order scheme. It is presented on Fig.13. Nevertheless, the third-order dissipation remains too high for an acceptable outcome at time 100. Again increasing order, we obtain a good result on Fig.14 with the fifth-order scheme. 

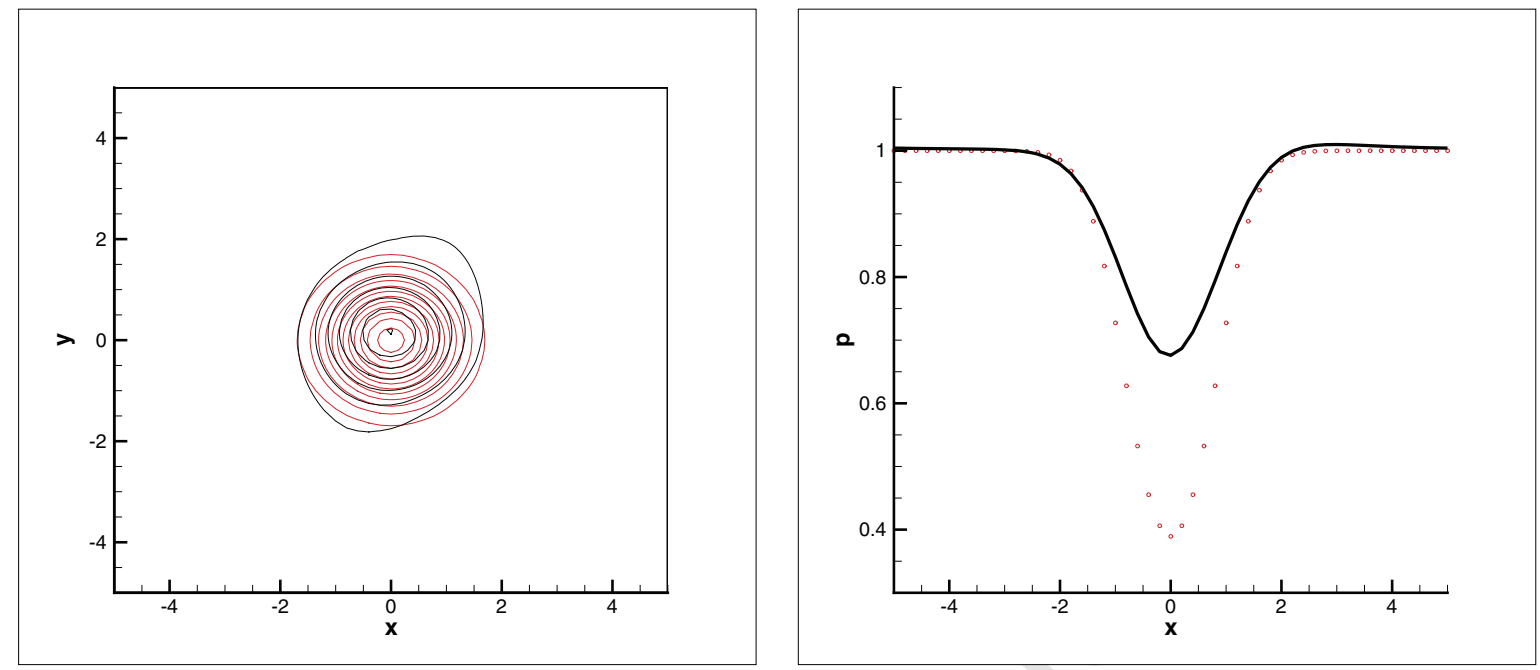

Figure 10: Advection of a vortex by $R B C_{2}-R K_{2} s d$ scheme on a $50 x 50$ mesh with periodicity conditions. Left: isobar lines (from $p=0.4$ to $p=0.95, \Delta p=0.05$ ) at $t=0,100$. Right: pressure cut on the axis $y=0$ at $t=0$ (symbols) and at $t=100$ (line).
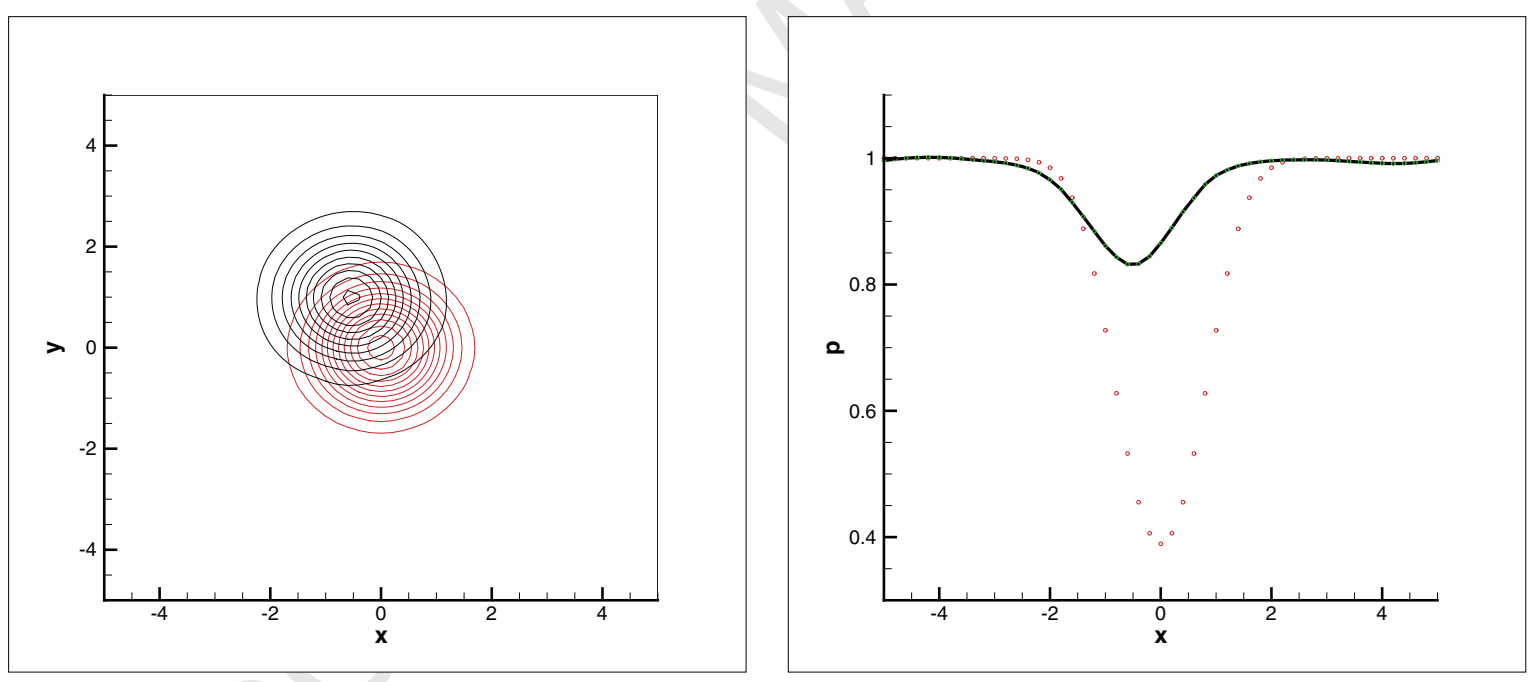

Figure 11: Advection of a vortex by $R B C_{3}-R K_{2} s d$ scheme on a $50 x 50$ mesh with periodicity conditions. Left: isobar lines (from $p=0.4$ to $p=0.95, \Delta p=0.05$ ) at $t=0,100$. Right: pressure cut on the axis $y=0$ at $t=0$ (symbols) and at $t=100$ (line). 

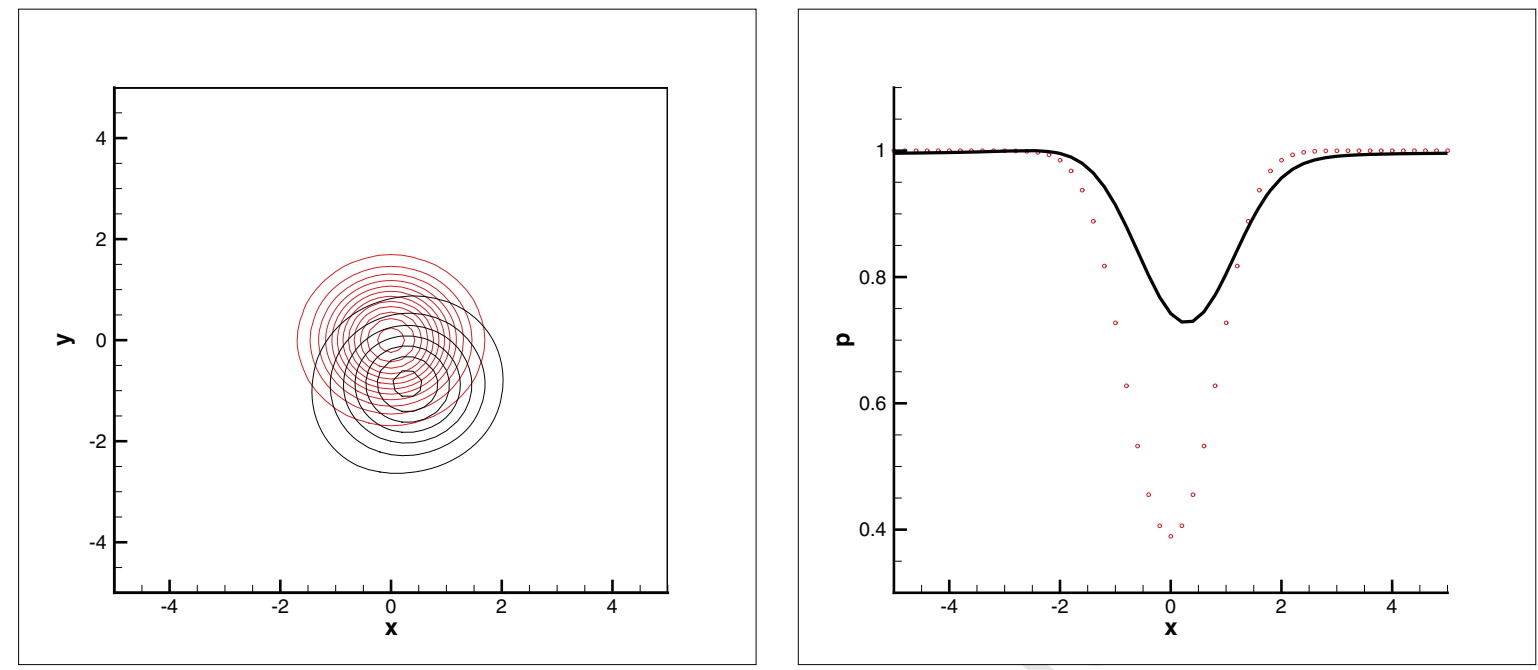

Figure 12: Advection of a vortex by $R B C_{2}-A B M_{2}\left(m_{f}=1\right)$ scheme on a $50 \times 50$ mesh with periodicity conditions. Left: isobar lines (from $p=0.4$ to $p=0.95, \Delta p=0.05$ ) at $t=0,100$. Right: pressure cut on the axis $y=0$ at $t=0$ (symbols) and at $t=100$ (line).
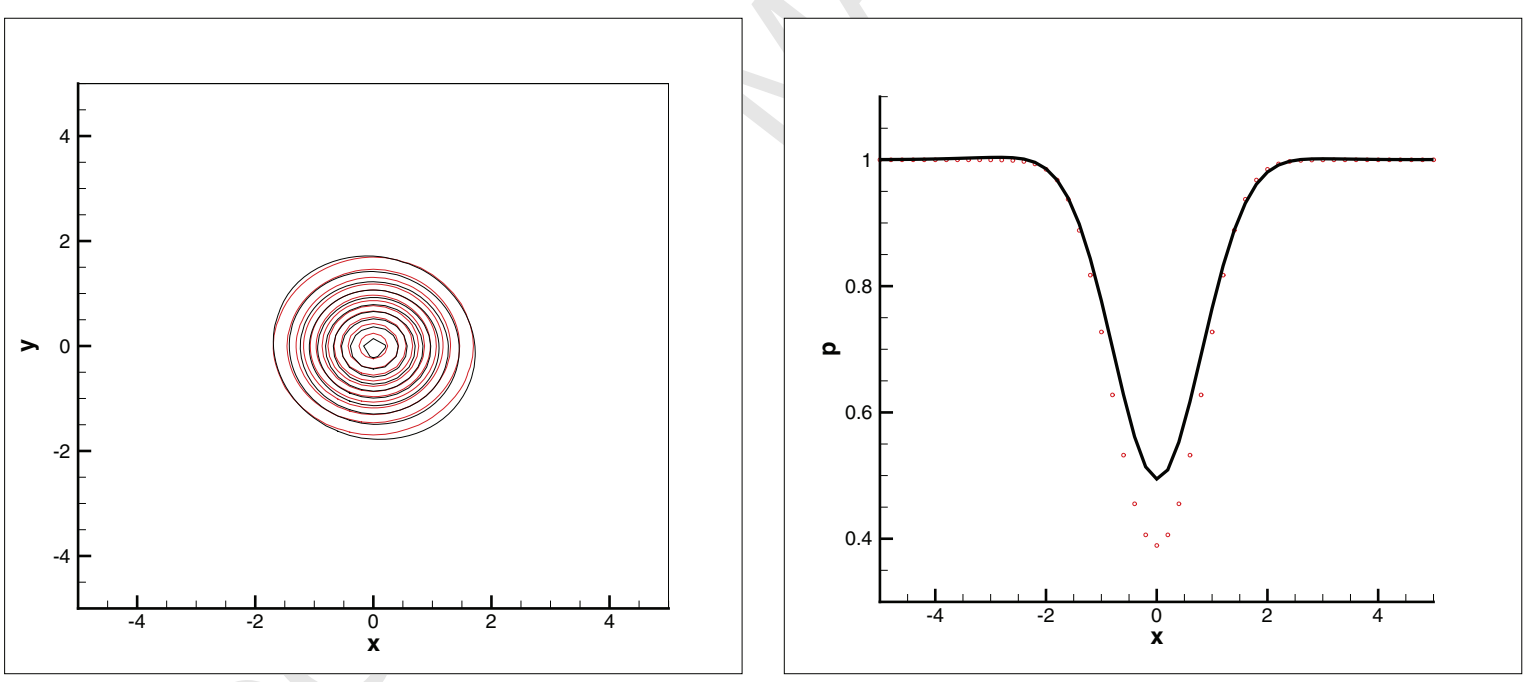

Figure 13: Advection of a vortex by $R B C_{3}-A B M_{3}\left(m_{f}=1\right)$ scheme on a $50 \times 50$ mesh with periodicity conditions. Left: isolines (from $p=0.4$ to $p=0.95, \Delta p=0.05$ ) at $t=0,100$. Right: pressure cut on the axis $y=0$ at $t=0$ (symbols) and at $t=100$ (line). 

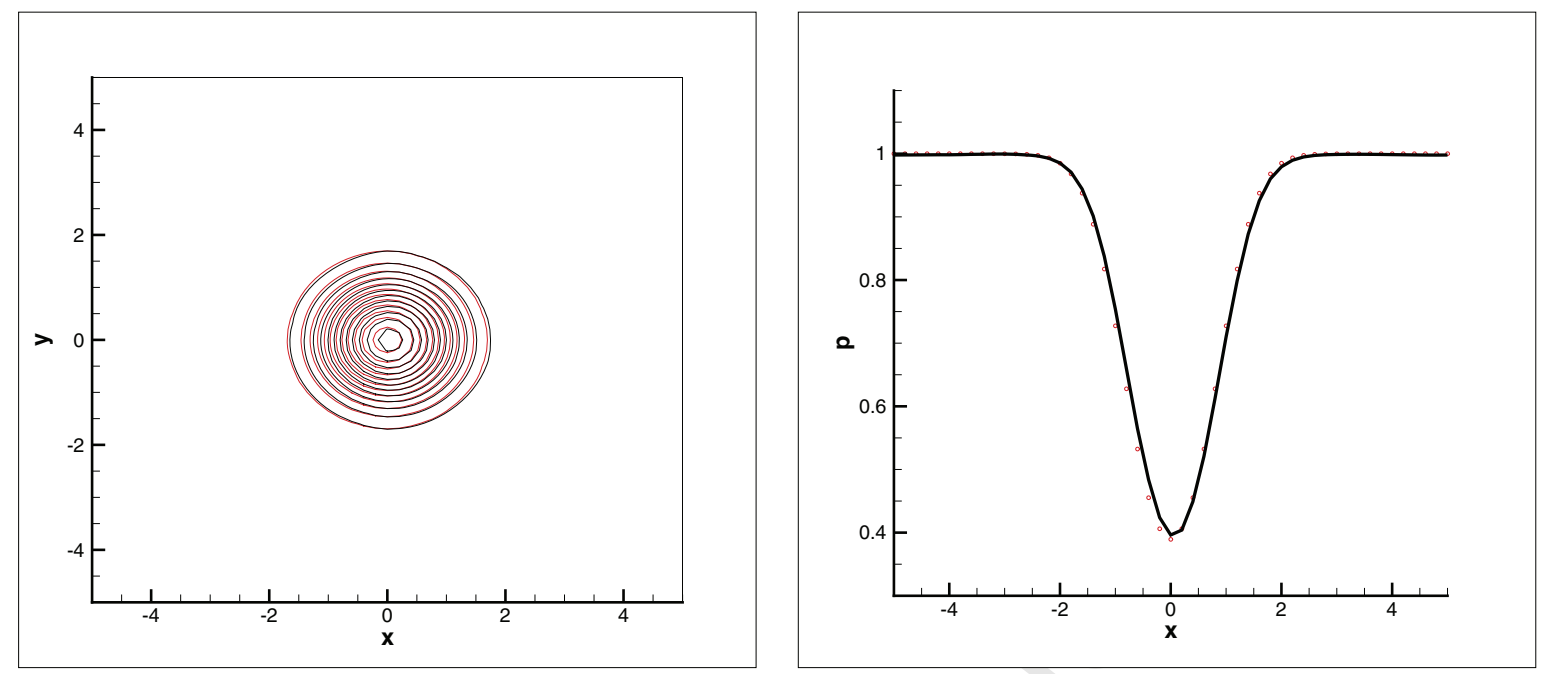

Figure 14: Advection of a vortex by $R B C_{5}-A B M_{4}\left(m_{f}=2\right)$ scheme on a $50 \times 50$ mesh with periodicity conditions. Left: isolines (from $p=0.4$ to $p=0.95, \Delta p=0.05$ ) at $t=0,100$. Right: pressure cut on the axis $y=0$ at $t=0$ (symbols) and at $t=100$ (line).

\subsection{Shock-vortex interaction}

Finally, we consider a moving homentropic vortex interacting with a steady shock. This problem was proposed by Jiang and Shu $[23]$. The space domain is here $[0,1]^{2}$ (it was $[0,2] \times[0,1]$ in the paper [23]). The vortex is initially located at $\left(x_{c}=0.25, y_{c}=0.5\right)$ and advected in the x-direction. The shock is positioned at $x=0.5$ and normal to the x-axis. Its upstream Mach number is $M_{0}=1.1$. The initial velocity components $u, v$ and the absolute temperature $T$ are defined upstream the shock in non-dimensional form as:

$$
\begin{gathered}
u=u_{0}+\epsilon Y \exp \left[\alpha\left(1-R^{2}\right)\right] \quad v=-\epsilon X \exp \left[\alpha\left(1-R^{2}\right)\right] \\
T=1-\frac{(\gamma-1) \epsilon^{2}}{4 \alpha \gamma} \exp \left[2 \alpha\left(1-R^{2}\right)\right]
\end{gathered}
$$

with

$$
X=\frac{x-x_{c}}{r_{c}}, \quad Y=\frac{y-y_{c}}{r_{c}}, \quad R^{2}=X^{2}+Y^{2}
$$

where $\epsilon=0.3$ (vortex strength), $\alpha=0.204$ (decay-rate control), $r_{c}=0.05$ (radius of maximun vortexstrength) and $u_{0}=M_{0} \sqrt{\gamma}$. The gas law is $p=\rho T$, with $\gamma=1.4$. Downstream the initial shock, the flow is uniform at the subsonic conditions behind the steady normal shock.

On the boundary the following conditions are applied: supersonic inflow on left side, subsonic outflow on right side and solid wall on lateral sides. Calculations are made on a 124x124 regular-mesh (contrary to [23] there is no x-refinement around the shock) until the time $t=0.35$ with a time-step $\Delta t=0.35 / 700$. Results obtained by the $R B C_{2}-A B M_{2}\left(m_{f}=1\right)$ scheme are shown on Fig.15. Fig.16 and 17 show the solutions given by the $R B C_{3}-A B M_{3}\left(m_{f}=1\right)$ and the $R B C_{5}-A B M_{4}\left(m_{f}=2\right)$ schemes in the same conditions. Due to the short vortex-excursion in this problem, the 3rd-order scheme produces a result close to the 5th-order one. These $R B C$ solutions are in good agreement with the $W E N O_{5}$ solution by Jiang and Shu [23] and the $R B C$ solutions published with Corre and Falissard in [4] using a time-formulation based on the Gear method solved by the dual-time stepping procedure. Unlike the $W E N O_{5}$ solution, the present results display some small oscillations (no kind of correction of the basic scheme), but the shock wave is spread over only two mesh-cells. Regarding the shock capturing, we note that the best results are obtained by $R B C_{3}-A B M_{3}\left(m_{f}=1\right)$. This is confirmed on Fig.18 by calculations with $R B C_{3}-A B M_{3}\left(m_{f}=1\right)$ 
and $R B C_{5}-A B M_{4}\left(m_{f}=2\right)$ on a finer grid $(248 \times 248$ mesh with $\Delta t=0.35 / 1400)$. Additional studies are needed to improve these shock-capturing properties.
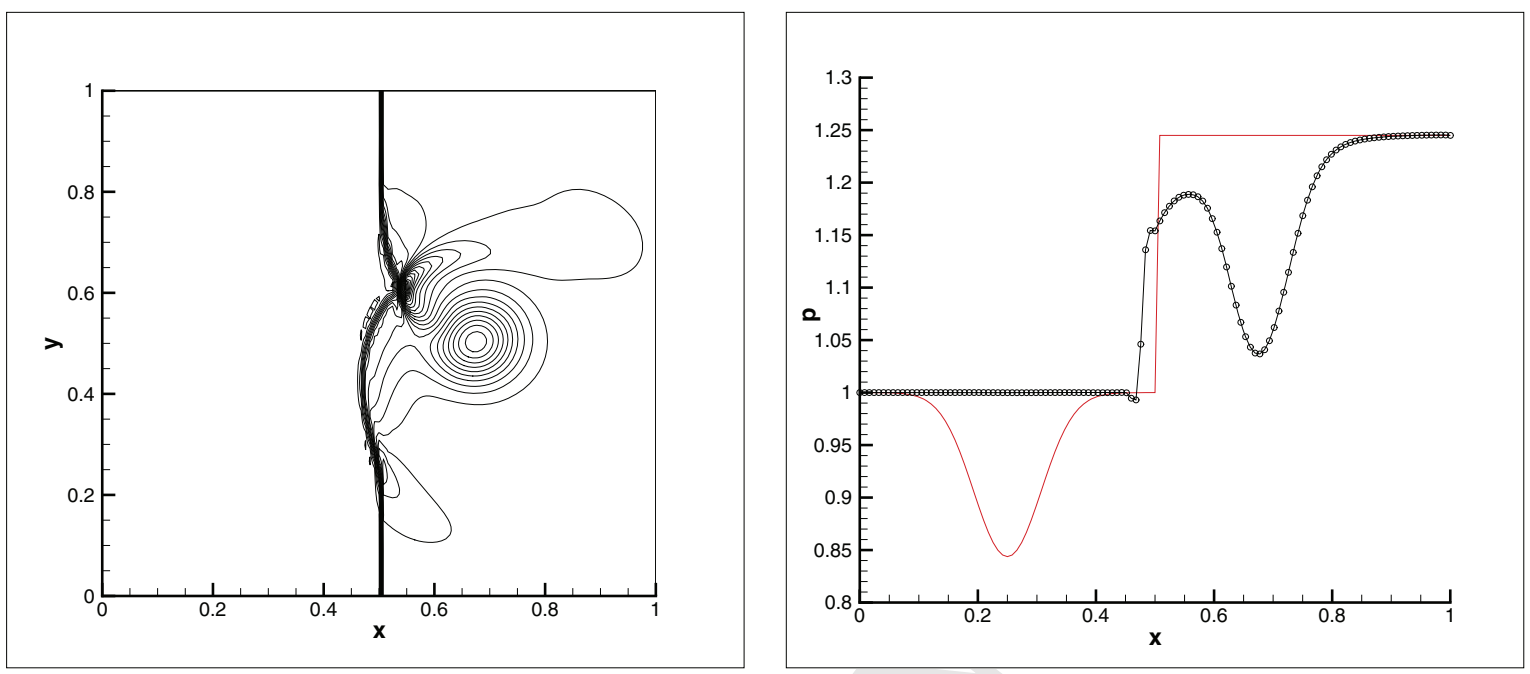

Figure 15: Shock-vortex interaction by $R B C_{2}-A B M_{2}\left(m_{f}=1\right)$ scheme on a $124 \times 124$ mesh. Left: isobar lines (from $p=0.79$ to $p=1.61, \Delta p=0.02)$ at $t=0$. Right: isobar lines at $t=0.35$.
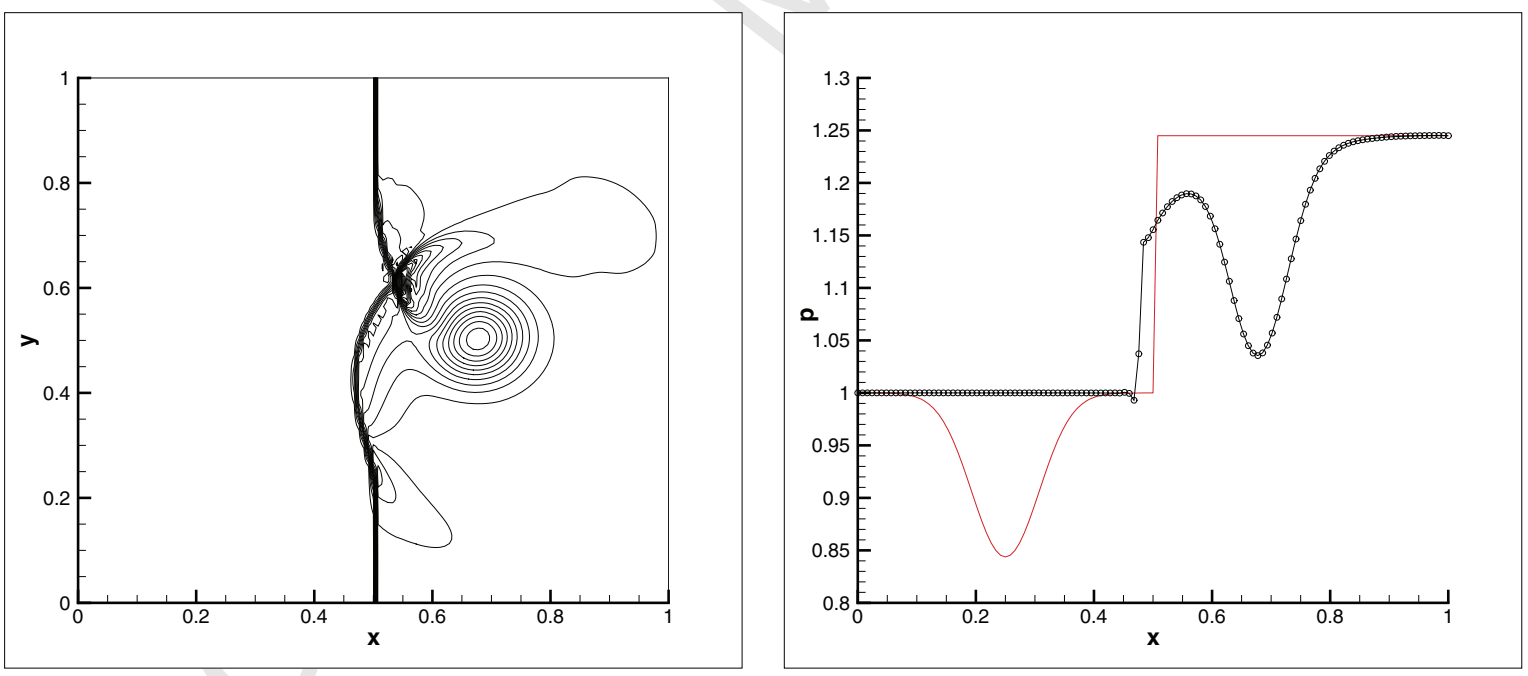

Figure 16: Shock-vortex interaction by $R B C_{3}-A B M_{3}\left(m_{f}=1\right)$ scheme on a $124 \mathrm{x} 124$ mesh. Left: isobar lines (from $p=0.79$ to $p=1.61, \Delta p=0.02$ ) at $t=0.35$. Right: pressure cut on $y=0.5$ at $t=0$. and at $t=0.35$ (line with mesh points). 

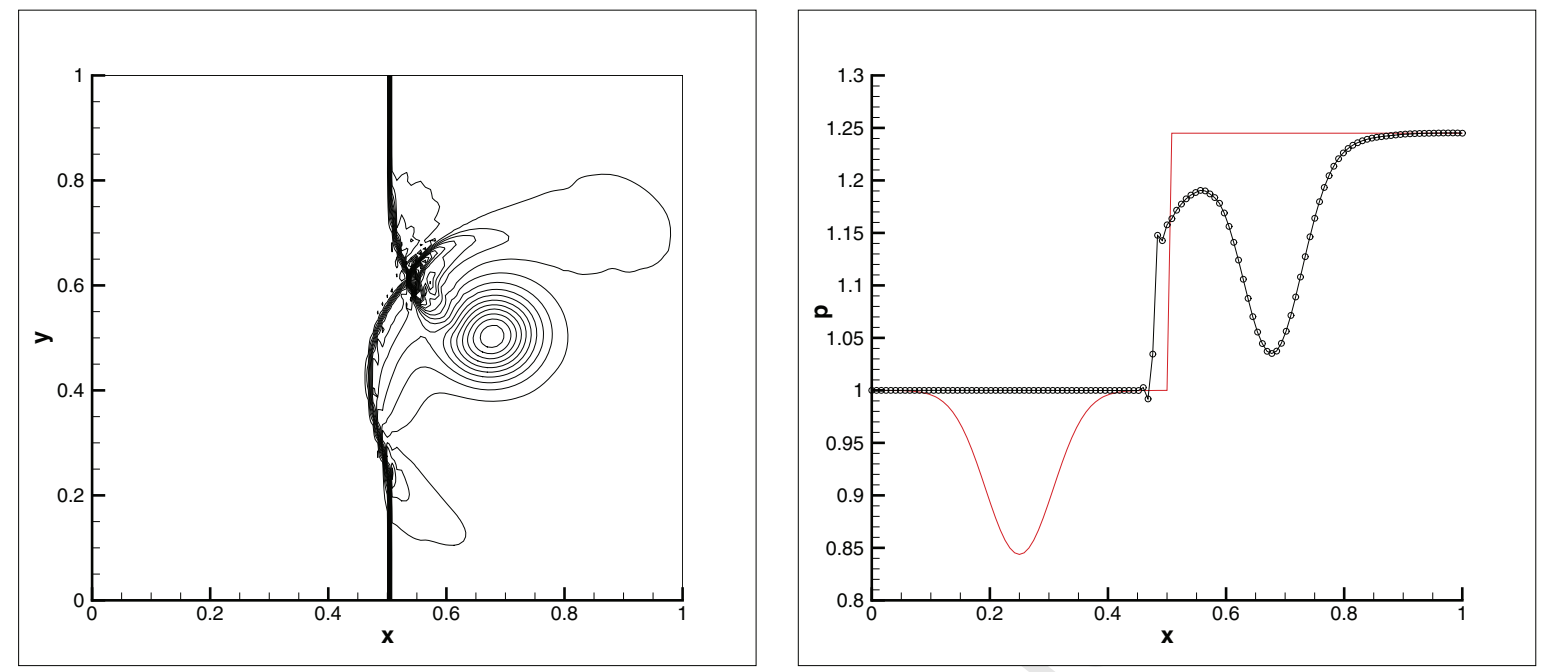

Figure 17: Shock-vortex interaction by $R B C_{5}-A B M_{4}\left(m_{f}=2\right)$ scheme on a $124 \times 124$ mesh. Left: isobar lines (from $p=0.79$ to $p=1.61, \Delta p=0.02$ ) at $t=0.35$. Right: pressure cut on $y=0.5$ at $t=0$. and at $t=0.35$ (line with mesh points).
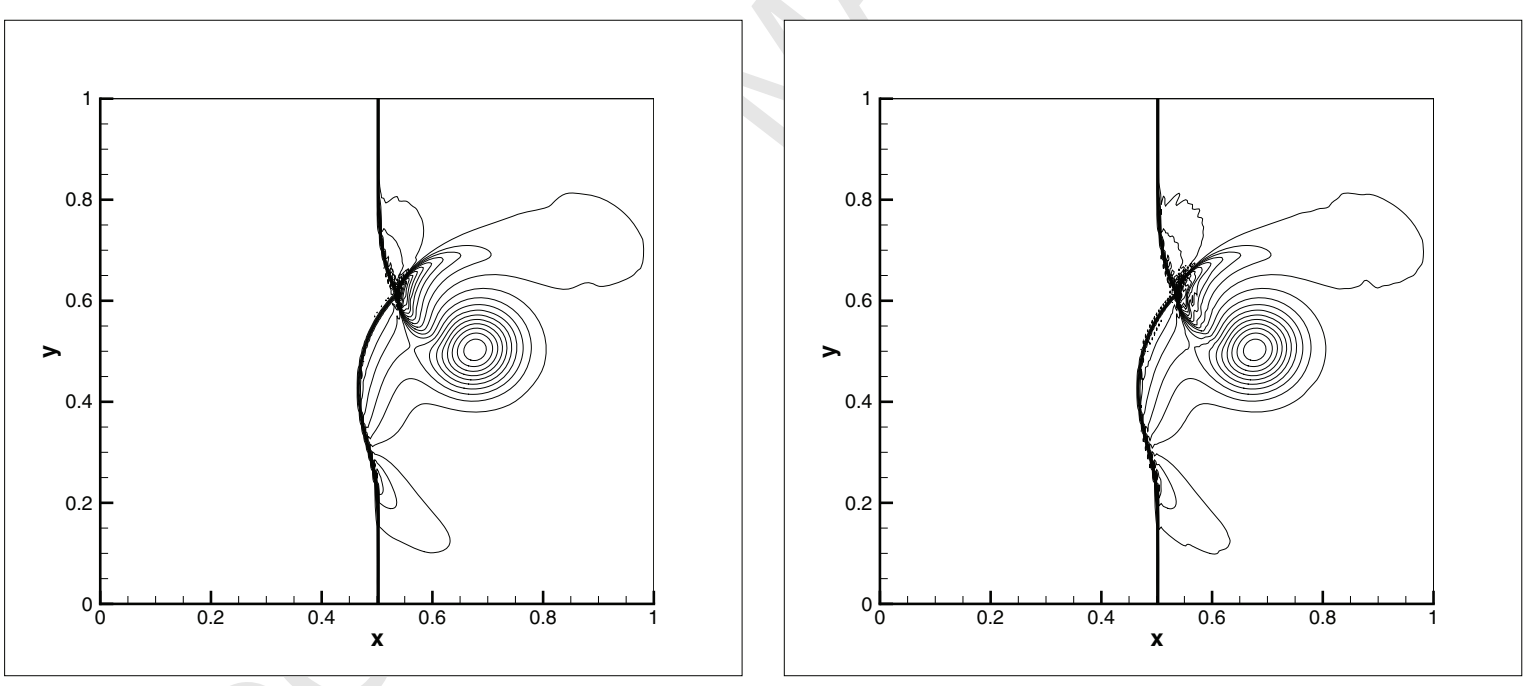

Figure 18: Shock-vortex interaction on a $248 \times 248$ mesh. Isobar lines (from $p=0.79$ to $p=1.61, \Delta p=0.02$ ) at $t=0.35$. Left: $R B C_{3}-A B M_{3}\left(m_{f}=1\right)$ scheme. Right: $R B C_{5}-A B M_{4}\left(m_{f}=2\right)$ scheme.

\section{Conclusion}

A new time-formulation for the residual-based compact schemes has been proposed in two-dimension. It is based on a space factorization of the terms containing the time-derivative without loss of spatial accuracy. The linear systems to be solved on the mesh lines for getting the time-derivative are block-tridiagonal for $R B C_{3}$ and $R B C_{5}$ and block-pentadiagonal for $R B C_{7}$. They are independent in a given direction and could 
therefore be fully parallelized in each direction. Numerical tests have shown the efficiency of the $R B C_{5}$ scheme associated with the $A B M_{4}$ time-integration.

Extension of the new time-formulation to three-dimension and to the compressible Navier-Stokes equations will be considered in a future study. First numerical results on the diagonal advection in a cubic domain of a Gaussian field with spherical symmetry show that the present space-factorization can be extended in 3-D without stability problem. Concerning the calculation of shock waves, it is planned to extend to the multidimensional case the correction inside a shock structure proposed for the one-dimensional Euler equations in [24] and inspired by an analysis of discrete shocks of high-order $R B C$ schemes applied to a scalar conservation law with a convex flux [25].

\section{References}

[1] A. Lerat, C. Corre, A residual-based compact scheme for the compressible Navier-Stokes equations, Journal of Computational Physics 170 (2001) 642-675.

[2] A. Lerat, C. Corre, Residual-based compact schemes for multidimensional hyperbolic systems of conservation laws, Computers \& Fluids 31 (2002) 639-661.

[3] A. Lerat, C. Corre, Higher order residual-based compact schemes on structured grids, 34th Comput. Fluid Dyn. Course, von Karman Institute for Fluid Dynamics, VKI LS 2006-1, pp. 1-111.

[4] C. Corre, F. Falissard, A. Lerat, High-order residual-based compact schemes for compressible inviscid flows, Computers and Fluids 36 (2007) 1567-1582.

[5] A. Lerat, K. Grimich, P. Cinnella, On the design of high order residual-based dissipation for unsteady compressible flows, Journal of Computational Physics 235 (2013) 32-51.

[6] K. Grimich, P. Cinnella, A. Lerat, Spectral properties of high-order residual-based compact schemes for unsteady comressible flows, Journal of Computational Physics 252 (2013) 142-162.

[7] M. Ricchiuto, A. Csik, H. Deconinck, Residual distribution for general time dependent conservation laws, Journal of Computational Physics 209 (2005) 249-289.

[8] R. Abgrall, Residual distribution schemes: Current status and future trends, Computers and Fluids 35 (2006) 641-669.

[9] H. Deconinck, M. Ricchiuto, Residual Distribution schemes: foundation and analysis, Encyclopedia of Computational Mechanics, John Wiley and Sons, 2007.

[10] R. Abgrall, A. Larat, M. Ricchiuto, C. Tavé, A simple construction of very high order non-oscillatory compact schemes on unstructured meshes, Computers and Fluids 38 (2009) 1314-1323.

[11] S. K. Lele, Compact finite difference schemes with spectral-like resolution, Journal of Computational Physics 103 (1992) $16-42$.

[12] B. Cockburn, C.-W. Shu, Nonlinear stable compact schemes for shock calculations, SIAM Journal of Numerical Analysis (1994) 607-627.

[13] H. C. Yee, Explicit and implicit multidimensional compact high-resolution shock-capturing methods: formulation, Journal of Computational Physics 131 (1997) 216-232.

[14] M. R. Visbal, D. V. Gaitonde, High-order accurate methods for complex unsteady subsonic flows, AIAA Journal 37 (1999) $1231-1239$.

[15] A. I. Tolstykh, High accuracy non-centered compact difference schemes for fluid dynamics applications, World Scientific, Singapore, 1994

[16] D. Fu, Y. Ma, A high order accurate difference scheme for complex flow fields, Journal of Computational Physics 134 (1997) $1-15$.

[17] C. Corre, G. Hanss, A. Lerat, A residual-based compact scheme for the unsteady compressible Navier-Stokes equations, Computers \& Fluids 34 (2005) 561-580.

[18] K. Grimich, B. Michel, P. Cinnella, A. Lerat, An accurate finite-volume formulation of a residual-based compact scheme for unsteady compressible flows, Computers and Fluids 92 (2014) 93-112.

[19] M.Ricchiuto, R.Abgrall, Explicit Runge-Kutta residual distribution schemes for time dependent problems: Second order case, Journal of Computational Physics 229 (2010) 5653-5691.

[20] MacCormack, Iterative modified approximate factorization, Computers and Fluids 30 (2001) 917-925.

[21] W. Hundsdorfer, S. Ruuth, R. Spiteri, Monotonicity-preserving linear multistep methods, SIAM Journal on Numerical Analysis 41 (2003) 605-623.

[22] J. D. Lambert, Numerical methods for ordinary differential systems: the initial value problem, J. Wiley edition, 1991.

[23] G. Jiang, C. Shu, Efficient implementation of weighted ENO schemes, Journal of Computational Physics 126 (1996) $202-228$

[24] A. Lerat, Shock capturing analysis of high-order schemes through analytical solution of the discrete schemes and their equivalent differential equations, in: International Conference on Computational Fluid Dynamics 8, Chengdu, China, ICCFD8-2014-198.

[25] A. Lerat, Steady discrete shocks of 5th and 7th-order RBC schemes and shock profiles of their equivalent differential equations, Journal of Computational Physics 272 (2014) 629-643. 